Review Article

\title{
Lamiaceae Essential Oils, Phytochemical Profile, Antioxidant, and Biological Activities
}

\author{
Luiz Renan Ramos da Silva, ${ }^{1}$ Oberdan Oliveira Ferreira, ${ }^{2,3}$ Jorddy Nevez Cruz ${ }^{(D)}{ }^{2}$ \\ Celeste de Jesus Pereira Franco, ${ }^{2}$ Tainá Oliveira dos Anjos, ${ }^{1,2}$ Marcia Moraes Cascaes, ${ }^{4}$ \\ Wanessa Almeida da Costa, ${ }^{5}$ Eloisa Helena de Aguiar Andrade, ${ }^{1,2,3,4}$ \\ and Mozaniel Santana de Oliveira $\mathbb{I D}^{1,2}$ \\ ${ }^{1}$ Programa de Pós-Graduação Em Ciências Biológicas-Botânica Tropical, \\ Universidade Federal Rural da Amazônia and Museu Paraense Emílio Goeldi, Av. Perimetral, 1901. Terra Firme, \\ Belém 66075-900, Pará, Brazil \\ ${ }^{2}$ Adolpho Ducke Laboratory-Botany Coordination, Museu Paraense Emílio Goeldi, Av. Perimetral, 1901, Terra Firme, \\ Belém 66077-830, Pará, Brazil \\ ${ }^{3}$ Postgraduate Program in Biodiversity and Biotechnology-Bionorte Network, \\ Universidade Federal do Pará-Rua Augusto Corrêa S/N, Guamá, Belém 66075-900, Pará, Brazil \\ ${ }^{4}$ Postgraduate Program in Chemistry-Universidade Federal do Pará-Rua Augusto Corrêa S/N, Guamá, \\ Belém 66075-900, Pará, Brazil \\ ${ }^{5}$ Universidade Federal do Pará-Rua Augusto Corrêa S/N, Guamá, Belém 66075-900, Pará, Brazil
}

Correspondence should be addressed to Mozaniel Santana de Oliveira; mozaniel.oliveira@yahoo.com.br

Received 17 September 2021; Accepted 25 November 2021; Published 14 December 2021

Academic Editor: Ângelo Luís

Copyright (c) 2021 Luiz Renan Ramos da Silva et al. This is an open access article distributed under the Creative Commons Attribution License, which permits unrestricted use, distribution, and reproduction in any medium, provided the original work is properly cited.

Medicinal and aromatic plants present important active compounds that have potential for use in food, pharmaceutical, and agriculture industries. In this sense, the present work aimed to conduct a literature review on the potential applications of essential oils from Lamiaceae species. Antioxidant, anti-inflammatory, and antimicrobial activities were evaluated. The importance of this study is demonstrated as a way to theoretically provide information on the use of different plants belonging to the Lamiaceae family, especially with regard to the physical, chemical, and biological properties of its essential oils.

\section{Introduction}

Several studies have shown that plants have bioactive compounds, such as terpenoids, alkaloids, glycosides, phytohormones, phenolic, and phenylpropanoids, that assist in the development of phytotherapeutic; in addition, natural products can be a viable alternative for the development of new drugs to control microorganisms resistant to traditional antibiotics [1-6]. Lamiaceae family has several species of aromatic plants that are applied in traditional medicine and in the pharmaceutical and food industries because of their biological properties [7]. They are used as stimulant for blood circulation and digestion, to strengthen the central nervous system, and as expectorant, antispasmodic, antiseptic [8], diuretic, carminative, and tonic [9]. The most popular plants in this family are oregano, rosemary, thyme, and sage [7].

The biological applications of the Lamiaceae are mainly related to its essential oils, which have various activities such as antioxidant, antitumor, anti-inflammatory, antiviral, analgesic, antitussive, antiasthmatic, antipyretic [9], antimicrobial, antiemetic, antifungal [10], insecticidal (against Aedes aegypti) [11], antidiabetic, antihypertensive [12], antipruritic, decongestant [13], antinociceptive [14], carminative, antirheumatic, antidepressant, neuroprotective, cholinergic [15], sedative, antiseptic, antiparasitic, 
anthelmintic, immunoregulatory [16], antiallergic [17], antiangiogenic, anti-hepatotoxic [18], anticancer [19], and others.

According to Pires et al. [20], medicinal plants began to be used both in traditional medicine (in natura) and in vegetal products, such as essential oils, enhancing the investigations of plant species and, consequently, their natural pharmacological agents, considering the different perspectives of rural and urban areas.

According to data from the WHO, more than $70 \%$ of the population uses herbal medicines as the main form of medication to treat diseases [21]. This growing interest for less industrialized products with functional ingredients stimulated the use of essential oils in several industrial sectors (food, cosmetics, hygiene, and agriculture), which are applied in product conservation and control of microorganisms [22-24]. The advantage of volatile oils over synthetic preservatives lies in the lower development of toxic side products and economic viability $[25,26]$.

The essential oils (EOs) are aromatic and volatile substances found in different plant parts (leaves, flowers, seeds, roots, and fruits) [26]. Also, according to the de Oliveira et al., [27], they are extracted by steam distillation, dry distillation, mechanical extraction, or other processes such as supercritical $\mathrm{CO}_{2}$ extraction. Essential oils are capable of undergoing physical processes which do not significantly alter their chemical compositions. The extraction methods vary according to the species, the plant part used, and the way the raw material is presented: fresh, partially dehydrated, or dried [28-30].

In general, EOs are formed by base elements (oxygen, hydrogen, and carbon), which generate aldehydes, esters, phenols, ketones, alcohols, organic acids, and substances with nitrogen/sulfur, hydrocarbons, and terpenes [31]. These functional groups are responsible for the properties of the oils. Those commonly found come from terpenoids and phenylterpenoids, with monoterpenes being the most frequent [32-34].

For the plant, EOs represent an adaptive advantage, being able to function as an attractant for pollination and as a natural defense against predator attacks [35]. The chemical composition of essential oils can vary within the same species because conditions such as cultivation site, collection method, environmental factors, and material storage can interfere in the production of metabolites [36].

The family to which plants belong can be important to make inferences about the composition of aromatic oils. For instance, Rutaceae family presents citrus species; Myrtaceae has as representative the eucalyptus; and Oleaceae, the jasmine [37-39]. Lamiaceae is one of the plant families that presents great interest regarding obtaining essential oils, which will be described in the next topics.

Thus, this paper is organized, besides introduction and final considerations, in three parts: (1) biological presentation and identification of Lamiaceae species; (2) chemical structures of biosynthesized molecules present in its EOs; and (3) different properties of these species.

\section{Lamiaceae Family}

The plant family Lamiaceae, formerly called Labiatae, for its flowers are characterized by a bilabiate corolla [40], Lamiaceae presents more than 7000 species that are grouped in about 240 genera; in Brazil, it is distributed in approximately 524 species belonging to 52 genera [41], and some of these species present aromatic properties, which confers great economic relevance to the Lamiaceae [42], being applied in cosmetics and herbal medicines. As examples of genera with such properties, Mentha, Ocimum, Salvia, Clerodendrum, and Plectranthus stand out.

Lamiaceae species are widely distributed around the globe, with various heights and habitats and greater abundance in the Mediterranean region [43]. They prefer hot areas; however, they can also be found in regions with low temperatures [41, 44]. In Brazil, Lamiaceae species are distributed throughout the country, with higher incidence in south, north, and northeast regions, comprising the Atlantic Forest, Amazon, and especially the Brazilian Cerrado [45-48].

The species of the Lamiaceae family have diversified morphological characteristics and may be herbs, herbaceous plants, shrubs, or tree species [41]. Nowadays, this is one of the biggest botanical families with flowers of different sizes, with warm and showy colors depending on the species. They are bisexual, with well-defined floral parts, apparent sepals and petals, inflorescence, and bilateral symmetry (zygomorphs), and the corolla tube is divided into two distinct parts, providing a "lip" shape, which is the main characteristic of the Labiatae family. Their leaves are normally simple, and their fruits are dry and multiple that become separated when ripe (schizocarpic fruits) $[40,49,50]$.

This family presents many species rich in flavonoids and terpenes, with diterpenoids being the most abundant [51]. They are also rich in other substances that in addition to providing medicinal use have also assisted in taxonomic classifications [52]. Among the spices with aromatic properties, the six best-known vernacular names are thyme, basil, oregano, rosemary, sage, and lemon balm [16]. This variety of bioactive compounds confers Lamiaceae properties such as antioxidant, insecticidal, fungicidal, and bactericidal [53], which can result in an aggregation of potential economic and pharmacological value.

\section{Lamiaceae Species Rich in Essential Oils}

Species of the Lamiaceae family produce large amounts of secondary metabolites, including the compounds present in essential oils in plants with biological activities and therapeutic potential $[41,44]$. Some examples include species $B$. officinalis, G. hederacea, $H$. pectinata. Lavandula. Lamium, M. officinalis, Mentha, M. vulgare, Origanum, Ocimum, $R$. officinalis, Salvia, S. hortensis, S. lavandulifolia, S. lateriflora, Sideritis, Teucrium, Thymus, and Ziziphora tenuior [54].

The genus Plectranthus is considered one of the richest in species diversity and essential oils, with monoterpenes and sesquiterpenes as the main constituents [55]. According to Crevelin et al. [56], the essential oils of Plectranthus 
neochilus and Plectranthus barbatus have antimicrobial effects against Streptococcus mutans. Besides antibacterial activity, Plectranthus also has antifungal action on Rhizopus stolonifer [57] and showed in vitro antischistosomal activity attributed to boldo essential oil, which exterminated $100 \%$ of Schistosoma mansoni adult worms [58]. It also caused reduction in female eggs of B-type Bemisia tabaci in tomatoes [59, 60]. Plectranthus amboinicus exhibited anti-inflammatory and good digestion activities, as well [61].

Among the herbaceous plants of the Lamiaceae family, the genus Ocimum is the most important due to its application in several areas [62], such as folk medicine, cooking, plant marketing, and perfumery industry [63]. Approximately 30 species compose this genus [63]. Among them, some are Ocimum gratissimum, O. basilicum L., $O$. micranthum, and O. campechianum. The extracts are applied in traditional medicine to treat rheumatism, epilepsy, some mental conditions, and respiratory tract infections [64-66]. Studies also have verified fungicidal, nematicidal, and larvicidal properties [67-70].

Additionally, the antifungal action of essential oils from Lamiaceae species has been used to improve food preservation. Isolated essential oils derived from thyme and oregano (thymol), clove (eugenol), and mint (menthol) were tested in strawberry preservation [71]. As a result, the treatment reduced strawberry degradation when compared with the control sample. Thymol oil showed better results, with a decay of $0 \%$ on day 1 to 20 on day 14 , with better results than the control sample, and the authors concluded that in addition to antimicrobial activity, treatment with essential oils also conferred antioxidant protection.

The essential oil of O. gratissimum L. was able to inhibit the growth of species such as Klebsiella sp., Pseudomonas aeruginosa, Escherichia coli, Staphylococcus aureus, and Salmonella enteritidis, even when used at different concentrations [72]. Pereira et al. [73], evaluated the antibacterial activity of O. gratissimum L., Cymbopogon citratus (DC) Stapf., and Salvia officinalis L. on microorganisms isolated from urinary tract infections. Salvia officinalis L. showed the best results, inhibiting the growth of more than $75 \%$ of all microorganisms evaluated. Species such as Salvia santolinifolia L, Salvia hydrangea L, Salvia mirzayanii $L$, Salvia triloba L, Salvia repens $L$, and Salvia runcinata $L$. also stand out.

The genus Hyptis (Hyptis ovalifolia Benth, Hyptis suaveolens $\mathrm{L}$, and Hyptis pectinata $\mathrm{L}$.) is predominant in the semiarid region of Northeastern Brazil, with prevalence of herbs but also with shrubby representatives and small trees [74]. Its EO has antiseptic, insecticidal, and fungicidal activities in addition to treating gastrointestinal infections and muscle pain $[72,75]$. In addition, the genus Perilla, whose main representative is Perilla frutescens L., has insecticidal activity, which is given by the isolated compound [76].

Genus Mentha, popularly known as mint, has menthol terpenes as main constituents of its essential oil. The greater yield is found in its leaves, presenting a considerable economic potential for food and pharmacological purposes $[77,78]$. This genus has a small diversity of plants all over the globe, containing only 25 species [79]. The biological activities presented by these Lamiaceae species are varied, e.g., antihypertensive, antioxidant, antimicrobial, antiallergic, biopesticidal, antitumor, anticancer, anti-inflammatory, and antiviral [80], which may be associated with the presence of compounds such as menthol, menthone, 1,8cineole, carvone, limonene, $\beta$-caryophyllene, and pulegone, among others [81].

Research on the genus Satureja L. reports that it is distributed in 30 species around the globe, and that it has beneficial properties for human health, such as in the treatment of pain caused by oxidative stress. Therefore, the essential oils from leaves and stems of Satureja spicigera L, Satureja cuneifólia L, and Satureja hortensis L. have compounds that work as antioxidants $[82,83]$.

Genus Thymus presents about 200 species. Most of them have antibacterial action due to the high content of phenolic compounds. T. caespititius, T. camphoratus, T. pectinatus Fihch, T. mastichina L., and Thymus vulgaris L. [84] are great examples, whereas $T$. numidicus and $T$. fontanesii have bactericidal activity [85].

\section{Chemical Composition of Lamiaceae Essential Oils}

Essential oils are volatile, lipophilic, and odoriferous substances produced by the secondary metabolism of plants. Due to their aromatic properties and chemical composition, they are used in cosmetics and folk medicine, with antiseptic, antifungal, and insecticidal actions [7].

In general, the biological activities that essential oils present are observed by the major substances present in their chemical composition. Their bioactivity is shown synergistically or by the isolated substances $[86,87]$.

In addition, the chemical composition of an essential oil can vary depending on the species, seasonality, circadian rhythms, plant age, and geographic location [36, 88]. As an example, the chemical profile of Hesperozygis myrtoides essential oil, which is a subshrub native to Cerrado and Atlantic Forest of Brazil, depends on altitude [89].

Essential oils are characterized by two or three major constituents. For instance, Mentha arvensis L. presented as major compounds menthol (86.1\%), menthone $(4.3 \%)$, and isomenthone (3.7\%) [90]; O. gratissimum L. showed as major constituents 1,8 -cineole (30.04\%), eugenol (27.58\%), and terpineol-4 (14.45\%) [91]; Origanum vulgare L. presented 4terpineol (18.4\%), sabinene hydrate (15.6\%), and thymol (13.6\%) [92]; and in the species Plectranthus ornatus Codd, the major compounds identified were $\alpha$-thujene (12.7-32.7\%), $\alpha$-pinene (5.5-23\%), sabinene $(7.51-17.8 \%), \beta$-pinene $(3.5-11.6 \%)$, 1-octen3-ol (0.6-11.1\%), 3-carene $(0.84-5.6 \%),(\mathrm{E})-\beta$-ocimene (1.5-8.4\%), $\alpha$-terpinyl acetate $(1.3-13.2 \%), \beta$-caryophyllene (3.9-13.6\%), and germacrene D (0.3-18.5\%) [93].

Giatropoulos et al. [94] evaluated 12 species of different plants of the Lamiaceae family and found high insecticidal action in the essential oils of T. vulgaris and O. vulgare. Such properties can be attributed to the high toxicity of its major constituents such as thymol (75.6\%), carvacrol (74.08\%), and p-cymene $(7.9 \%)$. The considerable toxicity of Satureja thymbra essential oil is also observed probably due to its 
major constituents carvacrol (32.4\%) and $\gamma$-terpinene (32.4\%).

Thus, the number of studies that seek applicability of the compounds present in Lamiaceae essential oils has increased since they have natural origin and present advantages when compared with synthetic substances [7]. Table 1 lists species rich in essential oils and their main constituents. Figures 1 and 2 show the main monoterpenes and sesquiterpenes identified in Lamiaceae essential oils.

\section{Antioxidant Activity}

Antioxidants are substances capable of retarding or preventing lipid oxidation caused by excessive oxygen radicals due to environmental factors or pathogens $[110,111]$. Such compounds, which can be natural or synthetic, have great importance in the food industry because they are used as preservatives in several products, delaying or preventing deterioration caused by the action of oxygen. Besides this, antioxidants have great relevance in biochemical and medical fields because they are able to neutralize the harmful effects of oxidation in animal tissues [112].

In recent years, there has been an increasing search for natural products with antioxidant properties due to the toxic side problems that synthetic products may cause [110]. Aromatic and medicinal plants are considered natural sources of antioxidant substances since their secondary metabolites act by inhibiting the formation of free radicals [113]. The aromatic and medicinal species of the Lamiaceae family have been constantly studied regarding their antioxidant activities, as shown in Table 2.

There are several techniques that determine the antioxidant capacity of essential oils and their components, among them are FRAP (ferric reducing antioxidant power), CUPRAC (cupric ion reducing antioxidant capacity), ABTS (2,20-azino-bis (3-ethylbenzothiazoline-6-sulfonic acid)), DPPH (2,2-diphenyl-1-picrylhydrazyl), quantification of products formed during lipid peroxidation (TBARS, LDL oxidation, and co-oxidation of $\beta$-carotene), and other methods [131].

The chemical composition and antioxidant activity of O. vulgare essential oil were studied by Morshedloo et al. [124]. After being analyzed by the DPPH method, all oils presented antioxidant activity, which was correlated with the high concentration of carvacrol. The essential oil from $O$. vulgare flowers showed the highest capacity to eliminate $\mathrm{DPPH}$ radicals $\left(\mathrm{EC}_{50}=0.68 \mathrm{~mL} / \mathrm{mL}\right)$, while the stem oil showed the lowest capacity $\left(\mathrm{EC}_{50}=1.82 \mathrm{~mL} / \mathrm{mL}\right)$. Regarding phenological stages, flowering showed the highest antioxidant activity $\left(\mathrm{EC}_{50}=0.86 \mathrm{~mL} / \mathrm{mL}\right)$ [123]. The authors also pointed out the strong antioxidant action of the essential oil from Origanum vulgare aerial parts using ABTS radical scavenging technique with $\mathrm{IC}_{50}=14.00 \mu \mathrm{g} / \mathrm{mL}$.

Besides O. vulgare, other species of the genus Origanum are reported in the literature to possess antioxidant activity. The essential oil of $O$. dictamnus flowers showed antioxidant activity by the DPPH method $\left(\mathrm{IC}_{50}=0.0459 \pm 0.0042 \%(\mathrm{v} / \mathrm{v})\right)$ that was attributed to its main compound, carvacrol [121]. The oils from aerial parts of $O$. floribundum were studied by
Hadjadj et al. [122] regarding their antioxidant potential by $\mathrm{DPPH}$ and ABTS assays. They presented better antioxidant activity by the ABTS method (33.6-95.5 $\mu \mathrm{g} / \mathrm{mL})$.

Zorzetto et al. [114] evaluated the antioxidant activity of Cedronella canariensis aerial parts using three methodologies (DPPH, ABTS, and FRAP). The authors demonstrated that the essential oil showed better antioxidant activity against the ABTS radical with $\mathrm{IC}_{50}=10.5 \mathrm{mg} / \mathrm{mL}$, which was about 20 times lower than Trolox. Although the DPPH method is similar to ABTS, C. canariensis oil presented low antioxidant activity $\left(\mathrm{IC}_{50}=615.5 \mathrm{mg} / \mathrm{mL}\right)$, about 500 times lower than Trolox. In addition, EOs of O. basilicum were shown to possess antioxidant activity by $\mathrm{DPPH}$ $\left(\mathrm{IC}_{50}=0.21-4.04 \mathrm{mg} / \mathrm{mL}\right)$ and $\beta$-carotene (bleaching content $=23.8-85.3 \%)$ [120].

Rosmarinus officinalis is known to possess several biological properties. Aerial parts of this species were collected in southeastern Anatolia (Turkey), and its essential oil showed antioxidant activity by DPPH and TBARS techniques with $\mathrm{IC}_{50}=10.08 \pm 0.15 \mu \mathrm{g} / \mathrm{mL}$ and $1.76 \pm 0.02 \mu \mathrm{g} / \mathrm{mL}$, respectively, which may be related to polyphenols and phenylpropanoids found in the oil [125]. Moghadam [126] showed that the essential oil of $R$. officinalis collected in Kermanshah (Iran) presented antioxidant activity, by DPPH assay $(13.00 \pm 0.51 \mu \mathrm{g} / \mathrm{mL})$, which was related to the presence of camphene and 1,8 cineole. Table 2 shows the relationship between Lamiaceae species and their antioxidant potential.

\section{Anti-Inflammatory Activity}

Inflammation is a sequential process produced by various biological stimuli, physical injuries, infectious agents, and antigen-antibody type interactions. Within the inflammatory process, there are reactive oxygen species (ROS) responses, which include superoxide anions, hydroxyl radicals, and hydrogen peroxides. These are released by activated macrophages, neutrophils, and dendritic cells [132].

The inflammatory process and its chain of development have presented relevance, and in this sense, intending to restrain this aggressive action on the organism, search for new anti-inflammatory agents, mainly of vegetable origin, is necessary [133]. It is worth emphasizing that within this branch, species such as Hyptis spicigera, which is used in folk medicine, have anti-inflammatory properties [134].

Essential oils from O. basilicum and O. gratissimum were obtained by hydrodistillation and hexane extraction. O. basilicum EO extracted by hydrodistillation presented eucalyptol and eugenol acetate, and the solvent extract presented 2-methylbenzyl and eugenol acetate. Regarding O. gratissimum EO, linalool, 1-terpinen-4-ol, alpha-caryophyllene, and trans-longipinocarveol were the major compounds. In this study, both EOs were analyzed for antiinflammatory potential on induced edema in rat ears, and the results showed that at doses of $50 \mu \mathrm{g} / \mathrm{ear}$, they exhibited significant anti-inflammatory effect $(p<0.05)$, with inhibitions of up to $80 \%$. According to the authors, these results were in accordance with the $100 \mu \mathrm{g} /$ ear hydrocortisone dose, which showed $54.8 \%$ of edema inhibition [118]. 
TABle 1: Chemical composition and yield of essential oils from Lamiaceae species.

\begin{tabular}{|c|c|c|c|c|}
\hline Species & Plant part & $\begin{array}{l}\text { Essential oil } \\
\text { yield }\end{array}$ & Main compounds & Reference \\
\hline Aeollanthus suaveolens Mart. ex Spreng & Leaves & $1.6 \%$ & $\begin{array}{l}\text { Massoia lactone, linalool, }(E) \text {-B-farnesene, } \\
\text { geraniol, 2,5-dimethoxy-p-cymene }\end{array}$ & {$[95]$} \\
\hline Calamintha nepeta (L.) Kuntze & Leaves & * & Pulegone, menthone, limonene & {$[96]$} \\
\hline $\begin{array}{l}\text { Clinopodium macrostemum (Moc. and } \\
\text { Sessé ex Benth.) Kuntze }\end{array}$ & Leaves & $0.80 \%$ & $\begin{array}{l}\text { linalool, nerol, caryophyllene, menthone, geranyl } \\
\text { acetate, terpineol, pulegone }\end{array}$ & {$[97]$} \\
\hline Hyptis dilatata Benth. & Leaves & * & $\begin{array}{c}\text { Fenchone, } 3 \text {-carene, } \alpha \text {-pinene, } \beta \text {-caryophyllene, } \\
\text { limonene, } \beta \text {-pinene, and camphor }\end{array}$ & {$[98]$} \\
\hline Hyptis martiusii Benth. & Leaves & $0.34 \%$ & $\begin{array}{c}\text { 1,8-cineole, d-carene, camphor, limonene, } \\
\text { germacrene B }\end{array}$ & [99] \\
\hline Lavandula dentata $\mathrm{L}$. & $\begin{array}{l}\text { Leaves and } \\
\text { stems }\end{array}$ & * & $\begin{array}{l}1,8 \text {-cineole, isolimonene, thuj-3-en-10-al, trans- } \\
\text { pinocarveol }\end{array}$ & {$[100]$} \\
\hline Melissa officinalis $\mathrm{L}$. & $\begin{array}{l}\text { Leaves and } \\
\text { flowers }\end{array}$ & $0.10 \%$ & $\begin{array}{l}\text { Citral, caryophyllene oxide, citronellal, geraniol, } \\
\text { geranyl acetate, } \beta \text {-caryophyllene }\end{array}$ & {$[101]$} \\
\hline Mentha arvensis $\mathrm{L}$. & $\begin{array}{l}\text { Leaves and } \\
\text { flowers }\end{array}$ & * & Citronellal and nerol & {$[102]$} \\
\hline M. piperita L & $\begin{array}{l}\text { Leaves and } \\
\text { flowers }\end{array}$ & * & $\begin{array}{c}\text { Menthone, menthol, pulegone and menthyl } \\
\text { acetate }\end{array}$ & {$[103]$} \\
\hline Minthostachys mollis (Benth.) Griseb. & Leaves & $0.98 \%$ & $\begin{array}{l}\text { Menthone, pulegone, cis-dihydrocarvone, } \\
\text { carvacryl acetate, linalyl acetate, and linalool }\end{array}$ & {$[104]$} \\
\hline Ocimum basilicum L. & Leaves & $1.56 \pm 0.15 \%$ & linalyl acetate and linalool & {$[105]$} \\
\hline O. gratissimum L. & Leaves & $*$ & 1,8-Cineole, eugenol, 4-terpineol & {$[91]$} \\
\hline O. gratissimum & $\begin{array}{l}\text { Leaves and } \\
\text { flowers }\end{array}$ & * & $\begin{array}{c}\text { Thymol, eugenol, 1,8-cineole, E-caryophyllene, } \beta \text { - } \\
\text { selinene }\end{array}$ & {$[106]$} \\
\hline Origanum scabrum Boiss. and Heldr. & Leaves & $1.5 \%$ & Carvacrol, thymol, p-cymene, $\gamma$-terpinene & {$[107]$} \\
\hline Origanum vulgare L. & Leaves & $*$ & 4-terpineol, sabinene hydrate, thymol & {$[92]$} \\
\hline Plectranthus amboinicus (Lour.) Spreng. & Leaves & $0.009 \%$ & Thymol, $\beta$-pinene, $\gamma$-terpinene, caryophyllene & {$[108]$} \\
\hline $\begin{array}{l}\text { Plectranthus barbatus var. grandis (L.H. } \\
\text { Cramer) Lukhoba and A.J. Paton }\end{array}$ & Leaves & $*$ & $\beta$-caryophyllene, $\alpha$-copaene, germacrene & {$[109]$} \\
\hline
\end{tabular}

(1)<smiles>CC(C)C12CCC(C)(CC1)C(=O)C2</smiles>

(7)<smiles>C=CC(C)(O)CCC=C(C)C</smiles>

(3)<smiles>CC(C)=C1CC[C@@H](C)CC1=O</smiles>

(5)<smiles>C=C(C)C1CC=C(C)CC1</smiles>

(10)

(6)

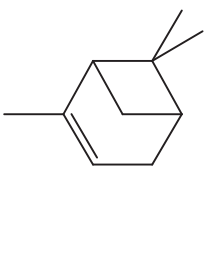

(11)<smiles>Cc1ccc(C(C)C)cc1O</smiles>

(13)<smiles>Cc1ccc(C(C)C)cc1</smiles><smiles>CC1C=C(O)C2(C(C)C)CC12</smiles>

(14)

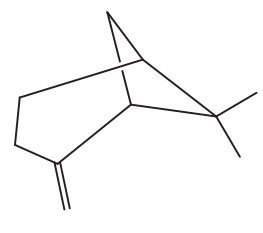

(15)<smiles>CC(C)=CCCC(C)CCO</smiles><smiles>Cc1ccc(C(C)C)c(O)c1</smiles><smiles>C=CC(=C)CCC=C(C)C</smiles>

Figure 1: Monoterpenes: $(1)=1,8$-cineole, $(2)=$ camphor, $(3)=$ pulegone, $(4)=$ borneol, $(5)=$ limonene, $(6)=\alpha$-pinene, $(7)=$ linalool, $(8)=$ carvacrol, $(9)=p$-cymene, $(10)=$ thujanol, $(11)=\beta$-pinene, $(12)=\alpha$-campholenal, $(13)=$ citronellol, $(14)=$ thymol, and $(15)=\beta$ myrcene. 
(1)

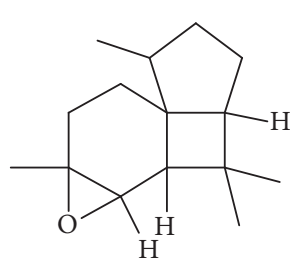

(5)
(2)<smiles>C[C@H]1CCC2=C(C[C@@H](C(C)(C)O)CC2)[C@@H]1C</smiles>

(3)<smiles>CC1=CC2(C(C)C)CCC(C)C(O)C2CC1</smiles>

(4)

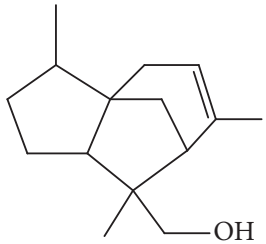

(8)<smiles>CC(=CCCC1(C)C2CC=C(C)C1C2)CO</smiles>

(6)<smiles>CC1=CCC2C3CCCC(C)(C3)C2C1</smiles><smiles>C=C(CO)C1CCC2(C)CCCC(=C)C2C1</smiles>

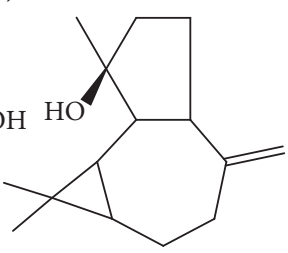

(9)

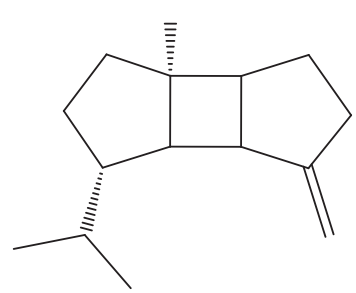

(10)

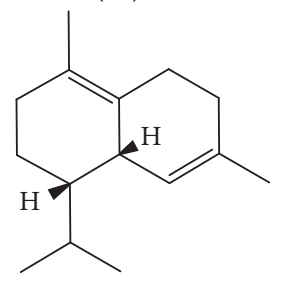

(11)

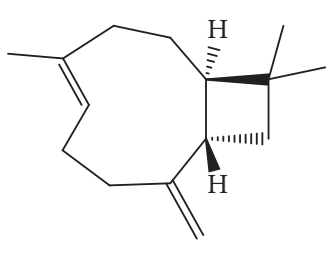

(12)

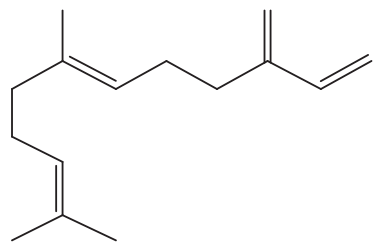

Figure 2: Sesquiterpenes: (1) = Italicene epoxide, (2) = guaiol, (3) = 1,10-di-epi-cubenol, (4) = 8-cedren-13-ol, (5) = (Z)- $\alpha$-trans-bergamotol, (6) $=\alpha$-copaene, $(7)=\beta$-costol, $(8)=$ spathulenol, $(9)=\beta$-bourbonene, $(10)=\delta$-cadinene, $(11)=\beta$-caryophyllene, and $(12)=\beta$-farnesene.

Six EOs from Lamiaceae family (Perilla frutescens, Mentha haplocalyx, Pogostemon cablin, $R$. officinalis, Lavandula angustifólia, and Scutellaria baicalensis) were studied regarding their anti-inflammatory potential. The major compounds found were linalool (0.05-46.55\%), $\alpha$-pinene (0.12-45.35\%), o-cymene (0.91-41.20\%), patchouli alcohol (28.27\%), dl-menthol (21.12\%), isobornyl acetate $(22.52 \%)$, D-limonene (0.01-18.42\%), $\alpha$-terpineol (0.07-4.88\%), and $\beta$-pinene $(0.08-2.03 \%)$. The anti-inflammatory tests were performed on the ears of rats of 6-8 weeks of age and bodyweight of $18 \pm 2 \mathrm{~g}$. They were induced by 12 -O-tetradecanoyl phorbol-13-acetate (TPA), and the drug ibuprofen was used as positive control. All six essential oils exhibited anti-inflammatory activity, and the essential oils isolated from $P$. cablin remarkably inhibited the formation of ear edema (29.87-81.25\% inhibition). Similarly, $R$. officinalis and Scutellaria baicalensis EOs worked better than ibuprofen (positive control) [135].

Thymol was the major compound found in Thymus vulgaris essential oil from two different regions of Algeria (Mostaganem-EO.TM and Tlemcen-EO.TT), with contents of $59.5 \%$ and $67.7 \%$, respectively. The anti-inflammatory activity was evaluated in vivo based on the inhibition of paw edema induced by carrageenan injection. As a result, both EO samples showed anti-inflammatory activities after 6 hours of administration $(400 \mathrm{mg} / \mathrm{kg})$, reducing paw edema by $58.4 \%$ for EO.TT and $50.4 \%$ for EO.TM [136]. In the study by Avola et al. [137], Origanum vulgare EO presented as major compounds carvacrol (35.95-0.22\%), thymol (25.2-0.27\%), p-cymene (21.54-0.35\%), and linalool
(4.26-0.05\%). This essential oil was tested to characterize the level of oxidative stress and evaluate the changes in intracellular ROS levels caused by IFN $\gamma$ and histamine in the presence or absence of $25 \mu \mathrm{g} / \mathrm{mL}$ of oil. Confluent NCTC 2544 cells were treated with H2DCFDA $72 \mathrm{~h}$ after stimulation. This ROS levels can cause inflammation-induced cellular damage. In this study, the results pointed out that cells pretreated with $O$. vulgare essential oil at $25 \mu \mathrm{g} / \mathrm{mL}$ or indomethacin at $10 \mu \mathrm{M}$ significantly reduced IFN $\gamma$ - and histamine-induced ROS production.

The chemical profile of Stachys lavandulifolia essential oil (EOSL) was characterized by the main compounds (-)- $\alpha$-bisabolol (56.4\%), bicyclogermacrene (5.3\%), $\delta$-cadinene $(4.2 \%)$, and spathulenol (2.9\%). And the anti-inflammatory activity of (-)- $\alpha$-bisabolol (BIS) and EOSL $(50 \mathrm{mg} / \mathrm{kg})$ was evaluated using carrageenan-induced inflammatory response in rats $(2 \%$ in $0.2 \mathrm{~mL})$. The results showed that both EOSL and BIS possessed significant inhibitory effects $(p<0.05$ or $p<0.01$ or $p<0.001)$ on different orofacial pain tests, but BIS proved to be more effective, significantly reducing nociceptive behavior in all tests [138].

\section{Antimicrobial Activity}

7.1. Antibacterial Activity. Lamiaceae family has great importance in the economic scenario, especially in the gastronomic sector, in which they are used as culinary herbs. Thus, there has been an incessant search for new antimicrobial agents from the secondary metabolism of plants [8], which according to Nieto (2017) can increase the shelf life of food products [7]. 
TABle 2: Antioxidant activity of essential oils from Lamiaceae species.

\begin{tabular}{|c|c|c|c|}
\hline Species and plant part & Technique & Results & Reference \\
\hline \multirow{3}{*}{ Cedronella canariensis (aerial parts) } & DPPH & $\mathrm{IC}_{50}=615.5 \pm 76.5 \mu \mathrm{g} / \mathrm{mL}$ & \multirow{3}{*}[114]{} \\
\hline & ABTS & $\mathrm{IC}_{50}=10.5 \pm 0.6 \mu \mathrm{g} / \mathrm{mL}$ & \\
\hline & FRAP & $\mathrm{IC}_{50}=3.8 \pm 1.46 \mu \mathrm{mol} \mathrm{TE} / \mathrm{g}$ & \\
\hline \multirow{2}{*}{ Mentha piperita (leaves) } & $\mathrm{DPPH}$ & Radical scavenging $=92.6 \pm 6.86 \%$ & \multirow{2}{*}[115]{} \\
\hline & Reducing power & Reducing power $=0.9 \pm 0.3$ & \\
\hline \multirow{2}{*}{ M. pulegium (aerial parts) } & DPPH & $\mathrm{IC}_{50}=321.41 \pm 2.53 \mu \mathrm{g} / \mathrm{mL}$ & \multirow{2}{*}[116]{} \\
\hline & FRAP & $\mathrm{IC}_{50}=58.27 \pm 2.72 \mu \mathrm{g} / \mathrm{mL}$ & \\
\hline \multirow{4}{*}{ M. rotundifolia (leaves) } & $\mathrm{DPPH}$ & $\mathrm{IC}_{50}=2222.2 \pm 25.2 \mu \mathrm{g} / \mathrm{mL}$ & \multirow{4}{*}[117]{} \\
\hline & ABTS & $\mathrm{IC}_{50}=133.8 \pm 4.8 \mu \mathrm{g} / \mathrm{mL}$ & \\
\hline & Reducing power & $\mathrm{IC}_{50}=166.6 \pm 1.9 \mu \mathrm{g} / \mathrm{mL}$ & \\
\hline & Phosphomolybdate & $\mathrm{IC}_{50}=45.2 \pm 1.2 \mu \mathrm{g} / \mathrm{mL}$ & \\
\hline \multirow{3}{*}{ M. spicata (aerial parts) } & $\mathrm{DPPH}$ & $\mathrm{IC}_{50}=3450 \pm 172.5 \mu \mathrm{g} / \mathrm{mL}$ & \multirow{3}{*}[118]{} \\
\hline & ABTS & $\mathrm{IC}_{50}=40.2 \pm 0.2 \mu \mathrm{g} / \mathrm{mL}$ & \\
\hline & FRAP & $\mathrm{IC}_{50}=215 \pm 4.50 \mu \mathrm{g} / \mathrm{mL}$ & \\
\hline M. spicata (leaves) & DPPH & $\mathrm{IC}_{50}=41,23 \mu \mathrm{g} / \mathrm{mL}$ & [119] \\
\hline \multirow{2}{*}{ O. basilicum (aerial parts) } & DPPH & $\mathrm{IC}_{50}=4.04 \pm 0.09-0.21 \pm 0.02 \mathrm{mg} / \mathrm{mL}$ & \multirow{2}{*}[120]{} \\
\hline & $\beta$-carotene & Bleaching $=23.8 \pm 0.6-85.3 \pm 1.0 \%$ & \\
\hline \multirow[t]{2}{*}{ Origanum dictamnus (flowers) } & DPPH & $\mathrm{IC}_{50}=0.0459 \pm 0.0042 \%(\mathrm{v} / \mathrm{v})$ & \multirow[t]{2}{*}[121]{} \\
\hline & $\mathrm{DPPH}$ & $\mathrm{IC}_{50}=369.9 \pm 3.1-1091.7 \pm 4.5 \mu \mathrm{g} / \mathrm{mL}$ & \\
\hline \multirow[t]{2}{*}{ O. floribundum (aerial parts) } & Reducing power & $\mathrm{IC}_{50}=230 \pm 5.2-315 \pm 3.9 \mu \mathrm{g} / \mathrm{mL}$ & \multirow[t]{2}{*}[122]{} \\
\hline & ABTS & $\mathrm{IC}_{50}=33.6 \pm 0.3-95.5 \pm 2.2 \mu \mathrm{g} / \mathrm{mL}$ & \\
\hline O. vulgare (aerial parts) & ABTS & $\mathrm{IC}_{50}=14,00257 \mathrm{mg} / \mathrm{mL}$ & {$[123]$} \\
\hline O. vulgare (flowers) & DPPH & $\mathrm{EC}_{50}=0.68 \mathrm{~mL} / \mathrm{mL}$ & {$[124]$} \\
\hline O. vulgare (stems) & $\mathrm{DPPH}$ & $\mathrm{EC}_{50}=1.82 \mathrm{~mL} / \mathrm{mL}$ & [124] \\
\hline \multirow{2}{*}{ R. officinalis (aerial parts) } & DPPH & $\mathrm{IC}_{50}=523.41 \pm 8.25 \mu \mathrm{g} / \mathrm{mL}$ & \multirow{2}{*}{ [116] } \\
\hline & FRAP & $\mathrm{IC}_{50}=85.74 \pm 7.57 \mu \mathrm{g} / \mathrm{mL}$ & \\
\hline \multirow{2}{*}{ R. officinalis (aerial parts) } & DPPH & $\mathrm{IC}_{50}=10.08 \pm 0.15 \mu \mathrm{g} / \mathrm{mL}$ & \multirow{2}{*}[125]{} \\
\hline & TBARS & $\mathrm{IC}_{50}=1.76 \pm 0.02 \mu \mathrm{g} / \mathrm{mL}$ & \\
\hline R. officinalis (aerial parts) & DPPH & $\mathrm{IC}_{50}=13.00 \pm 0.51 \mu \mathrm{g} / \mathrm{mL}$ & {$[126]$} \\
\hline \multirow[t]{2}{*}{ Satureja hortensis (aerial parts) } & DPPH & $\mathrm{IC}_{50}=13.45 \pm 0.35 \mu \mathrm{g} / \mathrm{mL}$ & \multirow[t]{2}{*}[127]{} \\
\hline & $\mathrm{DPPH}$ & $\mathrm{IC}_{50}=82.8 \pm 3.1 \mu \mathrm{g} / \mathrm{mL}$ & \\
\hline \multirow[t]{3}{*}{ Scutellaria immaculate (aerial parts) } & ABTS & $\mathrm{IC}_{50}=37.8 \pm 0.9 \mu \mathrm{g} / \mathrm{mL}$ & \multirow[t]{3}{*}[128]{} \\
\hline & FRAP & $\mathrm{IC}_{50}=720.19 \pm 4.8 \mu \mathrm{g} / \mathrm{mL}$ & \\
\hline & DPPH & $\mathrm{IC}_{50}=82.8 \pm 3.1 \mu \mathrm{g} / \mathrm{mL}$ & \\
\hline \multirow[t]{3}{*}{ S. ramosissima (aerial parts) } & ABTS & $\mathrm{IC}_{50}=93.6 \pm 0.8 \mu \mathrm{g} / \mathrm{mL}$ & {$[128]$} \\
\hline & FRAP & $\mathrm{IC}_{50}=837.23 \pm 3.2 \mu \mathrm{g} / \mathrm{mL}$ & \\
\hline & DPPH & $\mathrm{IC}_{50}=57.6 \pm 2.7 \mu \mathrm{g} / \mathrm{mL}$ & \\
\hline S. schachristanica (aerial parts) & ABTS & $\mathrm{IC}_{50}=66.6 \pm 1.2 \mu \mathrm{g} / \mathrm{mL}$ & {$[128]$} \\
\hline & FRAP & $\mathrm{IC}_{50}=779.64 \pm 8.6 \mu \mathrm{g} / \mathrm{mL}$ & \\
\hline Teucrium flavum (aerial parts) & DPPH & $\mathrm{IC}_{50}=31.5 \pm 1.8 \mu \mathrm{g} / \mathrm{mL}$ & {$[129]$} \\
\hline & DPPH & $\mathrm{IC}_{50}=0.619 \pm 0.11 \mu \mathrm{g} / \mathrm{mL}$ & \\
\hline Thymus capitatus (Leaves) & FRAP & $\mathrm{IC}_{50}=2,13 \pm 0.07 \mu \mathrm{g} / \mathrm{mL}$ & {$[130]$} \\
\hline & TAC & $\mathrm{IC}_{50}=0.78 \pm 0.14 \mu \mathrm{g} / \mathrm{mL}$ & \\
\hline
\end{tabular}

Essential oils from aerial parts (leaves) of Teucrium africanum and T. trifidum were characterized by the sesquiterpene hydrocarbons $\alpha$-cubebene and $\beta$-cubebene, respectively. In this study, they were evaluated for their antimicrobial potential. T. africanum EO showed minimum inhibitory concentration (MIC) equal to $0.16 \mathrm{mg} / \mathrm{mL}$ against Gram-positive bacterium Streptococcus pyogenes (ATCC 25923). Similarly, T. trifidum EO demonstrated remarkable antimicrobial activity with the MIC of $2 \mathrm{mg} / \mathrm{mL}$ against Gram-positive bacterium Staphylococcus aureus (ATCC 8668) [139].
In another study, the essential oil from the leaves and flowers of Origanum compactum, collected in six regions of Morocco was characterized by the major compounds carvacrol (2.18-63.65\%), p-cymene (6.69-42.64\%), and thymol (0.16-42.37\%). The antimicrobial activity of O. compactum EO was quite effective, being most active against Escherichia coli, Listeria innocua, and Staphylococcus aureus with inhibitory zones of $29.00 \pm 0.35 \mathrm{~mm}, 49.00 \pm 1.00 \mathrm{~mm}$, and $43.00 \pm 0.35 \mathrm{~mm}$, respectively [140].

The major compounds such as citronellal (14.40\%), isogeraniol (6.40\%), and geranyl acetate (10.20\%) 
TABLe 3: Antibacterial activity of Lamiaceae essential oils.

\begin{tabular}{|c|c|c|c|c|}
\hline Species & Bacteria & Method applied & Results & Reference \\
\hline \multirow{7}{*}{ Mentha spicata } & E. coli & & $11.8-21 \mathrm{~mm}$ & \multirow{7}{*}{ [119] } \\
\hline & S. enterica & & $8-18 \mathrm{~mm}$ & \\
\hline & P. aeruginosa & & $10-16 \mathrm{~mm}$ & \\
\hline & S. aureus & Disc-diffusion & $8-13 \mathrm{~mm}$ & \\
\hline & S. epidermidis & & $10.1-11.2 \mathrm{~mm}$ & \\
\hline & B. subtilis & & $9-11.5 \mathrm{~mm}$ & \\
\hline & $P$. aeruginosa & \multirow{4}{*}{ Agar-disc-diffusion } & $16.0 \pm 1.2 \mathrm{~mm}$ & \\
\hline \multirow{3}{*}{ Melissa officinalis } & K. pneumonia & & $3.0 \pm 0.6 \mathrm{~mm}$ & \multirow{3}{*}[141]{} \\
\hline & S. aureus & & $20.0 \pm 1.6 \mathrm{~mm}$ & \\
\hline & C. koseri & & $14.0 \pm 1.0 \mathrm{~mm}$ & \\
\hline \multirow{4}{*}{ Origanum compactum } & E. coli $\mathrm{K} 12$ & & $29.00 \pm 0.35 \mathrm{~mm}$ & \multirow{3}{*}{ [140] } \\
\hline & L. innocua 4030 & Microdilution & $49.00 \pm 1.00 \mathrm{~mm}$ & \\
\hline & S. aureus 25.923 & & $43.00 \pm 0,35 \mathrm{~mm}$ & \\
\hline & M. luteus & & $270 \mathrm{mg} / \mathrm{mL}$ & \multirow{4}{*}[142]{} \\
\hline \multirow{2}{*}{ O. vulgare } & S. aureus & Microdilution & $263 \mathrm{mg} / \mathrm{mL}$ & \\
\hline & E. coli & Mlicrodilution & $214 \mathrm{mg} / \mathrm{mL}$ & \\
\hline \multirow{12}{*}{ Salvia ringens } & P. aeruginosa & & $383 \mathrm{mg} / \mathrm{mL}$ & \\
\hline & E. coli & & 14.25 & \multirow{11}{*}[144]{} \\
\hline & S. typhimurium & & 14.25 & \\
\hline & S. enteritidis & & 11.40 & \\
\hline & P. tolaasii & & 14.25 & \\
\hline & P. aeruginosa & & 17.10 & \\
\hline & P. mirabilis & Microdilution & 17.10 & \\
\hline & S. aureus & & 9.50 & \\
\hline & B. cereus & & 9.50 & \\
\hline & M. flavus & & 9.50 & \\
\hline & S. lutea & & 11.40 & \\
\hline & L. monocytogenes & & 9.50 & \\
\hline Teucrium africanum & S. pyogenes (ATCC) & Microdilution & $0.16 \mathrm{mg} / \mathrm{mL}$ & \multirow{2}{*}{ [139] } \\
\hline T. trifidum & S. aureus & Microdilution & $2 \mathrm{mg} / \mathrm{mL}$ & \\
\hline \multirow{2}{*}{ Thymus pulegioides } & & Turbidity measurements & $0.5 \mathrm{mg} / \mathrm{mL}$ & \multirow{7}{*}{ [143] } \\
\hline & . & CFU & 27.500 bacterial $/ \mathrm{mL}$ & \\
\hline \multirow{2}{*}{ T. serpyllum } & S. mutans & Turbidity measurements & $0.9 \mathrm{mg} / \mathrm{mL}$ & \\
\hline & s. mutans & CFU & 1.750 .000 bacterial $/ \mathrm{mL}$ & \\
\hline T. vulgaris & S. mutans & $\begin{array}{l}\text { Turbidity measurements } \\
\text { CFU }\end{array}$ & $\begin{array}{c}0.75 \mathrm{mg} / \mathrm{mL} \\
3500 \text { bacterial } / \mathrm{mL}\end{array}$ & \\
\hline & & Turbidity measurements & $0.5 \mathrm{mg} / \mathrm{mL}$ & \\
\hline T. zygis & S. mutans & $\mathrm{CFU}$ & 4500 bacterial $/ \mathrm{mL}$ & \\
\hline
\end{tabular}

characterized the leaf essential oil of Melissa officinalis. It showed significant antimicrobial activity against Pseudomonas aeruginosa, Klebsiella pneumonia, Staphylococcus aureus, and Citrobacter koseri when compared with the conventional antibiotics cefaclor, oxacillin, and vancomycin [141].

Khan et al. [142] evaluated the chemical composition of the leaf essential oil of $O$. vulgare, which presented carvacrol $(70.2 \pm 1.37 \%)$ and $\gamma$-terpinene $(5.6 \pm 0.11 \%)$. In this study, O. vulgare EO was evaluated for its antimicrobial potential against Gram-positive (Micrococcus luteus and Staphylococcus aureus) and Gram-negative (Escherichia coli and Pseudomonas aeruginosa) bacteria in comparison with its purified compound carvacrol. The results showed that carvacrol was more effective and completely inhibited the growth of E. coli at $200 \mathrm{mg} / \mathrm{mL}$ and also retarded the growth of $P$. aeruginosa, with $\mathrm{IC}_{50}$ value of $151 \mathrm{mg} / \mathrm{mL}$. The essential oil, on the other hand, inhibited bacterial growth at concentrations of $270,263,214$, and $383 \mathrm{mg} / \mathrm{mL}$ for $M$. luteus, $S$. aureus, E. coli, and $P$. aeruginosa, respectively.

In the study conducted by Niksic et al. [119], major compounds carvone $(56,4 \%)$, limonene $(16,2 \%), 1,8$-cineole (7\%), $\beta$-pinene $(2,4)$, and $\alpha$-terpinene $(2,3 \%)$ characterized Mentha spicata essential oil. It exhibited significant bactericidal activity against both Gram-positive and Gram-negative microorganisms, with $M$. spicata essential oil being more sensitive and showing greater zone of inhibition against Escherichia coli $(11.8-21 \mathrm{~mm})$, Salmonella enterica (8-18 mm), and Pseudomonas aeruginosa (10-16 mm). Gram-positive bacteria, on the other hand, showed moderate antimicrobial activity at concentrations of $1 \%, 5 \%$, and $10 \%$, against $S$. aureus $(8-13 \mathrm{~mm})$, Staphylococcus epidermidis (10.1-11.2 mm), and Bacillus subtilis (9-11.5 mm). According to the authors, Mentha spicata antibacterial 


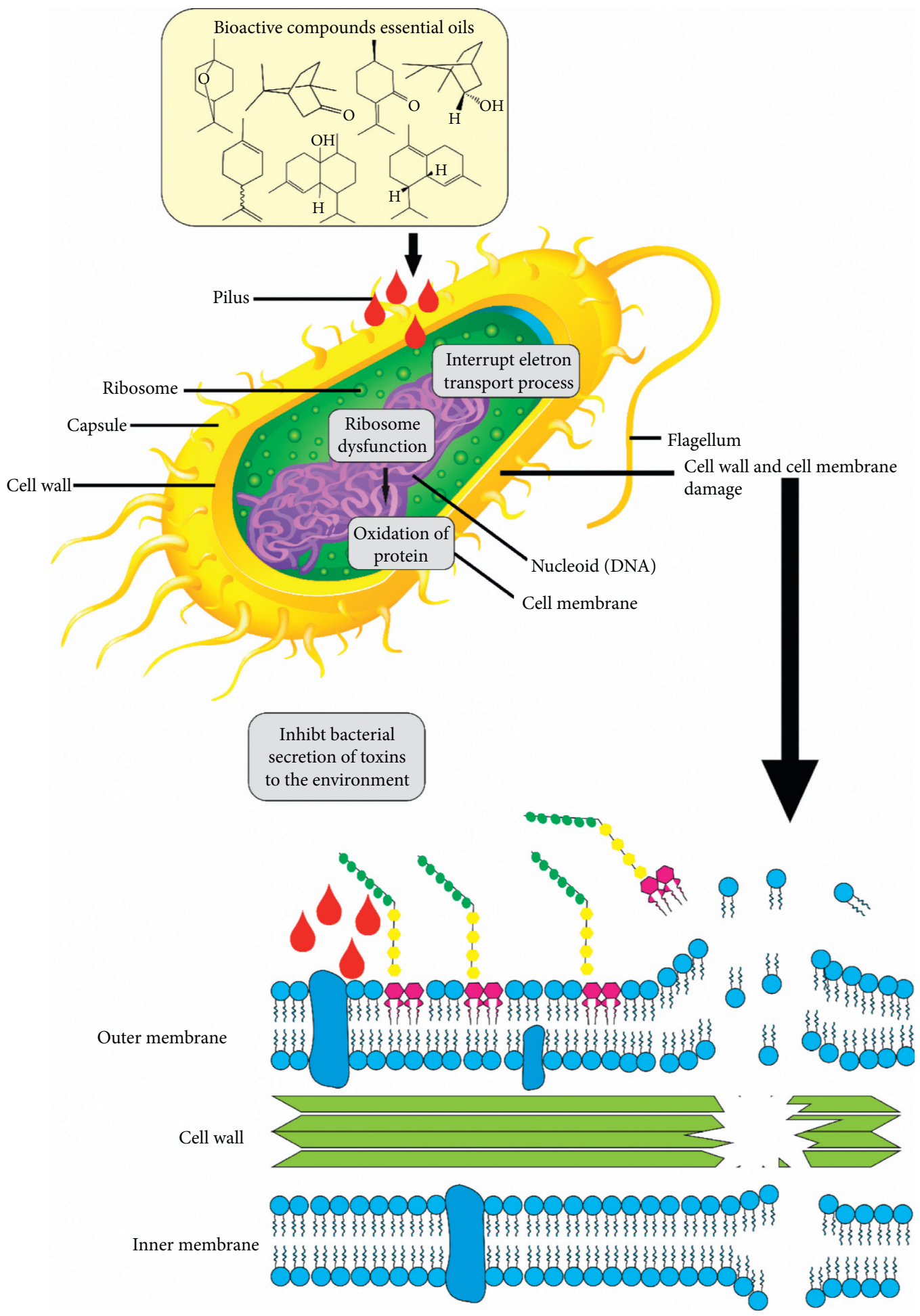

FIgURe 3: Potential mechanism of action of essential oils on bacteria, adapted from $[145,146]$.

activity can be attributed to the presence of several chemical groups, such as oxygenated monoterpenes and hydrocarbons, which favors the use of $M$. spicata essential oil as an antiseptic agent in the pharmaceutical and food industries.
The essential oils of four species of the genus Thymus (T. vulgaris, T. zygis, T. serpyllum, and T. pulegioides) were analyzed, and their chemical profile was characterized by oxygenated monoterpenes and thymol, which 
TABLe 4: Antifungal activity of Lamiaceae essential oils.

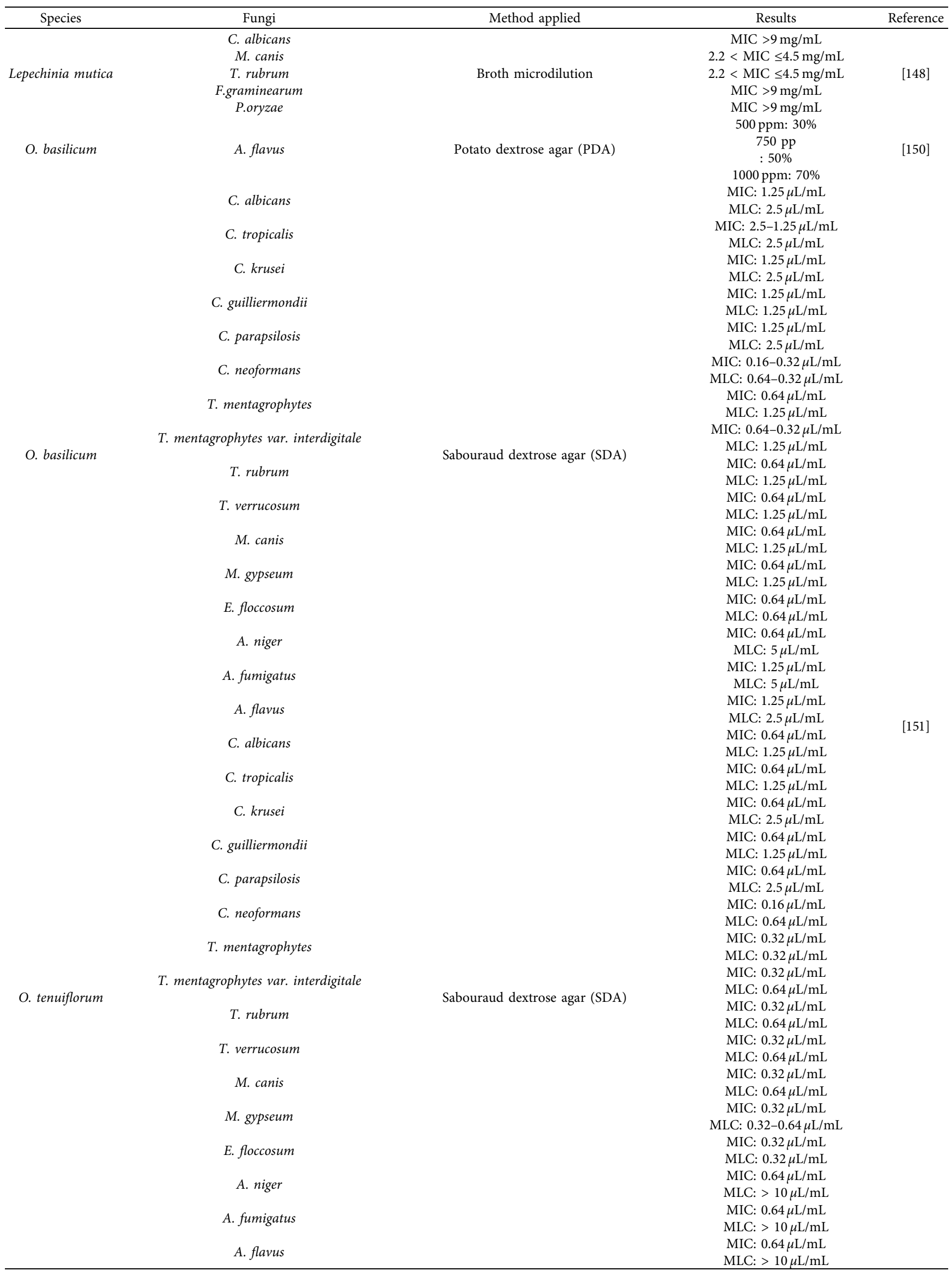


TABLE 4: Continued.

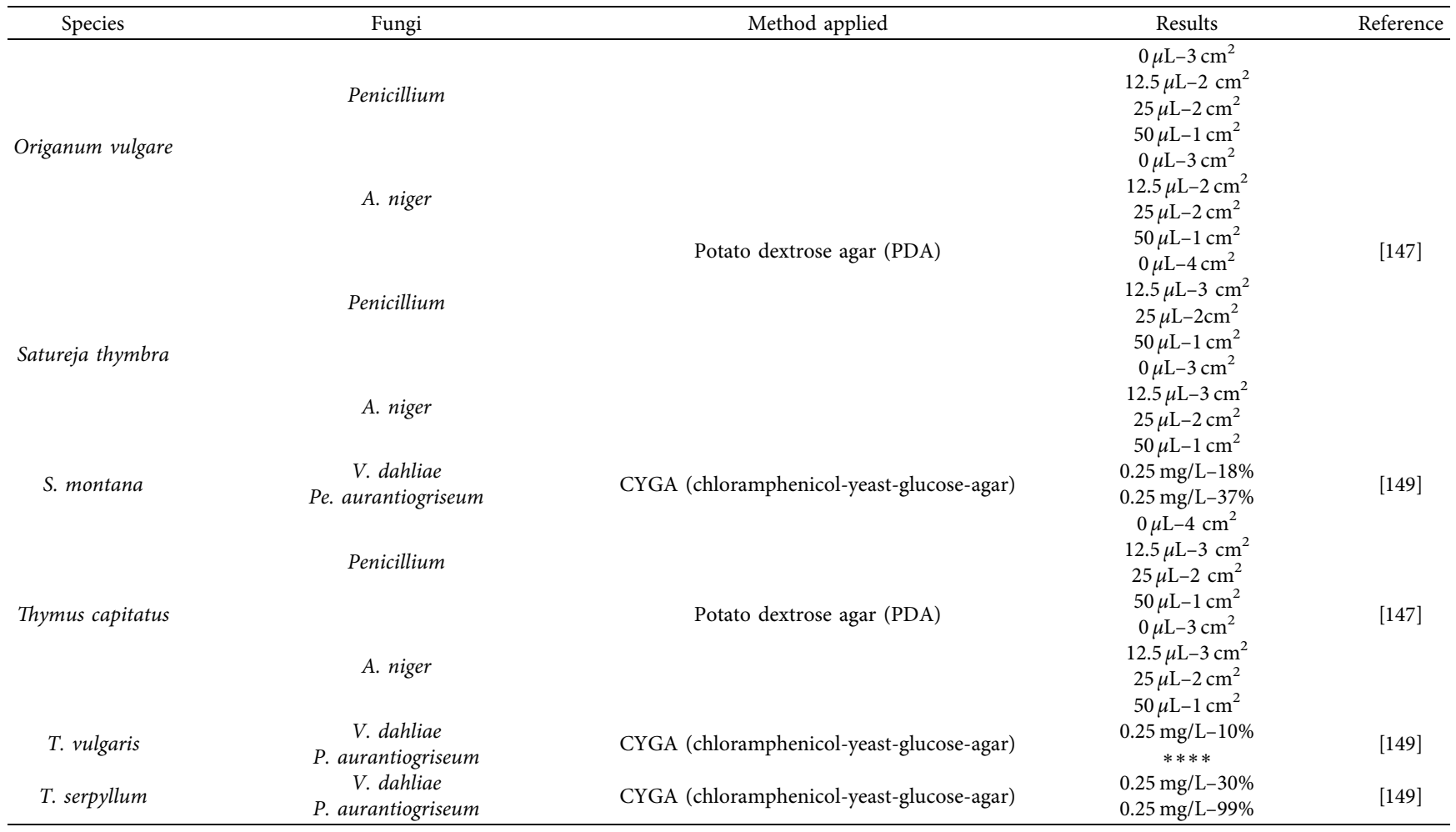

was the major chemical constituent. It presented the following contents: $37.7 \%, 41.7 \%, 13.7 \%$, and $44.5 \%$ for T. vulgaris, T. zygis, T. serpyllum, and T. pulegioides, respectively. The determination of their antibacterial activity against the Gram-positive bacterium Streptococcus mutans was performed by turbidity measurement, determination of colony-forming units (CFUs), and the live/dead staining method. In the turbidity test, essential oils of T. zygis and T. Pulegioides had the highest minimum inhibitory concentration (MIC equal to $0.5 \mathrm{mg} / \mathrm{mL}$ ), followed by $T$. vulgaris (MIC $=0.75 \mathrm{mg} / \mathrm{mL}$ ) and T. serpyllum $(\mathrm{MIC}=0.9 \mathrm{mg} / \mathrm{mL})$. Regarding CFU, all four essential oils significantly affected S. mutans growth. The lowest CFU value was found for T. serpyllum (1,750 CFU [bacterial $/ \mathrm{ml}])$, followed by $T$. vulgaris $(3,500 \mathrm{CFU}$ [bacterial $/ \mathrm{ml}]), T$. zygis $(4,500 \mathrm{CFU}$ [bacterial $/ \mathrm{ml}])$, and T. pulegioides $(27,500 \mathrm{CFU}$ [bacterial/ml]). Regarding the live/dead staining method, T. vulgaris essential oil had the strongest in vitro antimicrobial activity against S. mutans, followed by $T$. pulegioides and $T$. serpyllum. In contrast, the essential oil of T. zygis had the weakest effect [143].

Leaf essential oil of Salvia ringens was characterized by 1.8-cineole (31.99\%), camphene (17.06\%), borneol (11.94\%), and $\alpha$-pinene $(11.52 \%)$. It was tested against six Gramnegative bacteria: E. coli (ATCC25922), Salmonella typhimurium (ATCC14028), Salmonella enteritidis (ATCC13076), Pseudomonas tolaasii (NCTC387), Pseudomonas aeruginosa (ATCC27853), and Proteus mirabilis (ATCC14273), and five Gram-positive bacteria: Staphylococcus aureus (ATCC25923), Bacillus cereus (ATCC10876), Micrococcus flavus (ATCC14452), Sarcina lutea
(ATCC10054), and Listeria monocytogenes (ATCC15313). The results showed that $S$. ringens EO showed the strongest antibacterial activity with MIC equal to $9.50-17.10 \mathrm{mg} / \mathrm{mL}$ [144]. In Table 3, results of the antibacterial activity of Lamiaceae essential oils are shown. Figure 3 shows a probable mechanism of action of essential oils in bacteria.

7.2. Antifungal Activity. Fungal infections can be very dangerous for humans, especially when it concerns food, because fungi have the ability to produce mycotoxins and also reduce or destroy the nutritional value of grains during storage. Thus, it is important to mention the numerous studies with Lamiaceae essential oils with antimicrobial properties against fungi [7]. EOs of O. vulgare, Thymus capitatos, and Satureja thymbra were analyzed and showed the following major constituents: carvacrol (82.48\%), p-cymene $(5.00 \%)$, and $\gamma$-terpinene $(2.62 \%)$. They were tested against two phytopathogenic fungi (Aspergillus Niger and Penicillium spp.) isolated from slices of bread left outdoors at room temperature. Results showed that the addition of essential oils had significant effect $(p<0.05)$ on decreasing their colony surface area. Thus, oregano (O. vulgare), thyme (Thymus capitatus), and pink savory (S. thymbra) can be incorporated into bread recipes and be used in the food industry, as they have antimicrobial properties [147].

In the study by Niksic et al. [148], Lepechinia mutica EO was characterized by shyobunol $(10.80 \%)$, 3-carene $(8.69 \%)$, $\delta$-cadinene $(6.96 \%)$, and globulol $(5.91 \%)$, and it was tested against three serious human pathogenic fungi: Candida 


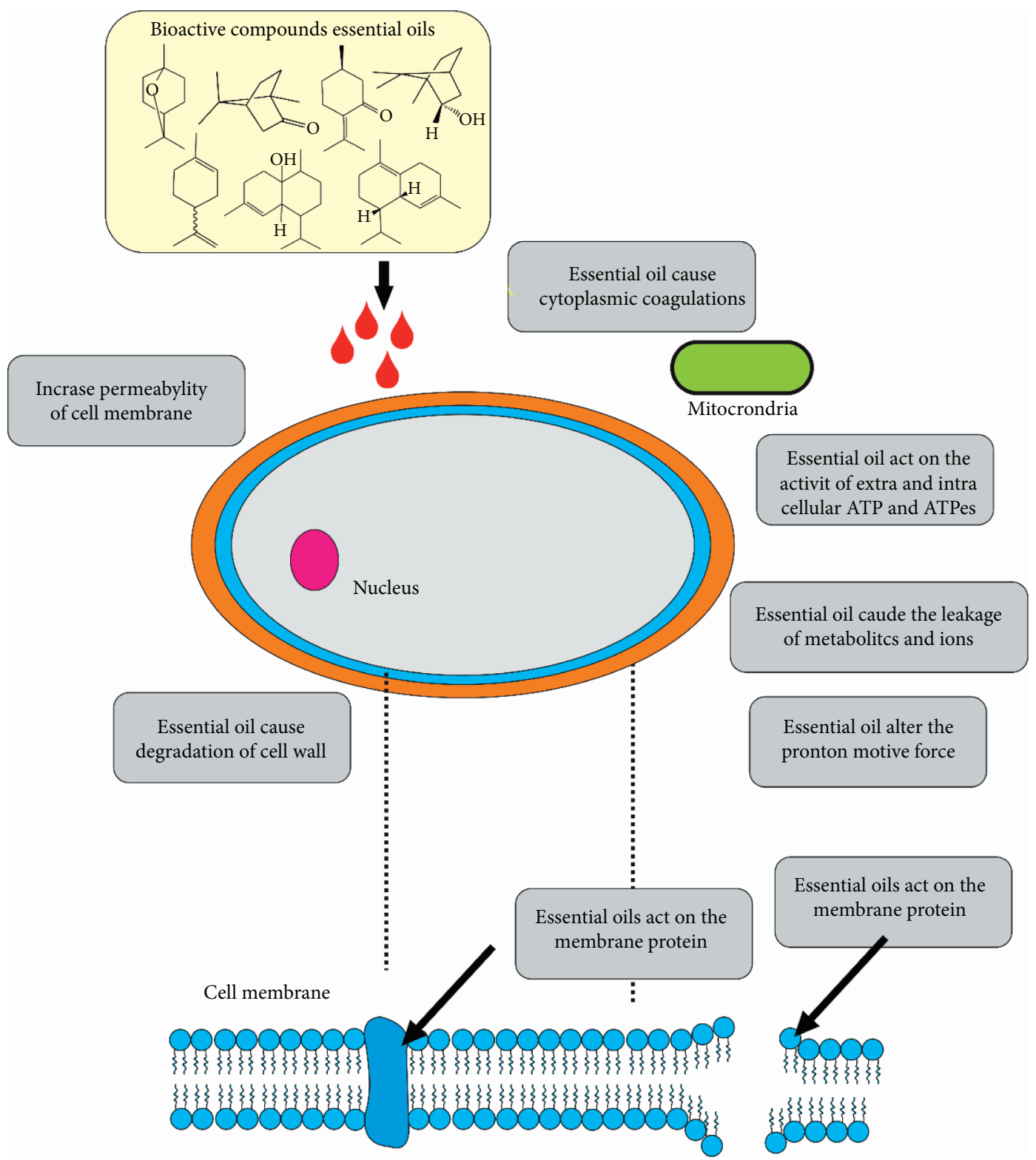

Figure 4: Potential mechanism of action of essential oils in fungi, adapted from [152].

albicans, Trichophyton rubrum, and Microsporum canis, and two potent plant pathogens: Pyricularia oryzae and Fusarium graminearum. Compared with the positive controls amphotericin B and voriconazole, L. mutica EO exhibited moderate activity against $M$. canis and T. rubrum, having MIC values ranging from 2.2 to $4.5 \mathrm{mg} / \mathrm{mL}$.

Rus et al. [149] evaluated the chemical composition of EOs from three species of the Lamiaceae family (T. vulgaris, T. serpyllum, and Satureja montana), which showed the following major compounds: $\mathrm{p}$-cymene, $\gamma$-terpinene, and carvacrol. Their antifungal activity was evaluated against Verticillium dahliae and Penicillium aurantiogriseum at concentrations ranging from $0.25-15 \mathrm{mg} / \mathrm{L}$. The essential oils of T. vulgaris, T. serpyllum, and Satureja montana exhibited mycelial growth inhibition (MGI) equal to $10 \%$,
$30 \%$, and $18 \%$, respectively, against $V$. dahlia, and $0 \%, 99 \%$, and $37 \%$ against $P$. aurantiogriseum at $0.25 \mathrm{mg} / \mathrm{L}$. At the other concentrations, growth was almost nonexisting. These results show that $T$. vulgaris EO was the most effective, especially against $P$. aurantiogriseum, which was highly sensitive.

The major compounds linalool (48.4\%), 1,8-cineole (12.2\%), eugenol (6.6\%), methyl cinnamate (6.2\%), $\alpha$-cubebene $(5.7 \%)$, caryophyllene $(2.5 \%), \beta$-ocimene $(2.1 \%)$, and $\alpha$-farnesene $(2.0 \%)$ characterized the chemical profile of the essential oil from $O$. basilicum leaves. It was tested against Aspergillus flavus at concentrations of 500, 750, and $1000 \mathrm{ppm}$, and the results showed that at $500 \mathrm{ppm}$, this EO showed inhibition rate of $30 \%$; at $750 \mathrm{ppm}, 50 \%$; and at $1000 \mathrm{ppm}, 70 \%$. These results are promising for curing 
mycotic infections and as a pharmaceutical preservative against $A$. flavus growth. It may also be used for aflatoxin B1 production [150].

Ocimum tenuiflorum essential oil was characterized by methyl eugenol (84.7\%) and $\beta$-caryophyllene (7.4\%), whereas $O$. basilicum EO had its chemical profile characterized by the major constituents linalool (35.1\%), eugenol (20.7\%), and 1,8-cineole (9.9\%). In this study, they were tested against C. albicans, C. tropicalis, C. krusei, C. guilliermondii, C. parapsilosis, Cryptococcus neoformans, Trichophyton mentagrophytes, T. mentagrophytes var. interdigitale, Trichophyton rubrum, T. verrucosum, Microsporum canis, M. gypseum, Epidermophyton floccosum, Aspergillus niger, A. fumigatus, and A. flavus, with significant results. The essential oil of $O$. tenuiflorum exhibited prominent antifungal activity against $C$. neoformans $(\mathrm{MIC}=0.16 \mu \mathrm{L} / \mathrm{mL})$ and dermatophyte fungi $(0.32 \mu \mathrm{L} / \mathrm{mL})$. However, it had no fungicidal effect against Aspergillus niger (MLC> $10 \mu \mathrm{L} / \mathrm{mL}$ ), while O. basilicum EO hindered the development of this kind of fungus, presenting fungicidal activity (MLC $=2.5-5 \mu \mathrm{L} / \mathrm{mL})$ and $\mathrm{MIC}$ equal to $0.64-1.25 \mu \mathrm{L} / \mathrm{mL}$ [151]. In Table 4 , the antifungal activity of Lamiaceae essential oils is shown. Figure 4 shows the possible mechanism of action of essential oils on fungi.

\section{Conclusion}

Lamiaceae species and, consequently, their essential oils may have peculiarities according to their cultivation system, climate, and location. Thus, some chemical and biological properties tend to change, showing great potential, especially regarding medicinal applications.

They have been used to fight certain diseases due to their antioxidant, antifungal, antibacterial, and anti-inflammatory actions. Additionally, there are other Lamiaceae species that act against insects, as well as on environmental remediation (phytoremediation) and thermal protection (green roofs).

Because this botanical family is extremely versatile, more studies on its compounds must be conducted since it has great pharmacological potential, with a promising future. Therefore, this review contributes to future studies on Lamiaceae and encourages the use of alternative natural resources for different purposes.

\section{Data Availability}

The datasets generated and analyzed during the current study are available in the databases, such as PubMed, Google Scholar, Web of Science, Scopus, and Science Direct (datasets can be requested from the corresponding author upon formal request).

\section{Conflicts of Interest}

The authors declare that there are no conflicts of interest.

\section{Acknowledgments}

Dr. Mozaniel Santana de Oliveira thanks PCI-MCTI/MPEG as well as $\mathrm{CNPq}$ for the scholarship granted (process number: 302050/2021-3).

\section{References}

[1] E. Jang, K.-S. Inn, Y. P. Jang, K.-T. Lee, and J.-H. Lee, "Phytotherapeutic activities of sanguisorba officinalis and its chemical constituents: a review," The American Journal of Chinese Medicine, vol. 46, no. 02, pp. 299-318, 2018.

[2] M. Din, F. Ali, A. Waris, F. Zia, and M. Ali, "Retracted: phytotherapeutic options for the treatment of COVID-19: a concise viewpoint," Phytotherapy Research, vol. 34, no. 10, pp. 2431-2437, 2020.

[3] P. S. X. Yap, B. C. Yiap, H. C. Ping, and S. H. E. Lim, "Essential oils, A new horizon in combating bacterial antibiotic resistance," The Open Microbiology Journal, vol. 8, no. 1, pp. 6-14, 2014.

[4] S. Monteiro and C. L. C. Brandelli, Farmacobotânica: Aspectos Teóricos e Aplicação, Farm. Asp. Teóricos e Apl., 2017.

[5] S. G. Silva, M. S. de Oliveira, and J. N. Cruz, "Supercritical CO2 extraction to obtain Lippia thymoides Mart. \& Schauer (Verbenaceae) essential oil rich in thymol and evaluation of its antimicrobial activity," The Journal of Supercritical Fluids, vol. 168, Article ID 105064, 2021.

[6] O. Sticher, "Natural product isolation," Natural Product Reports, vol. 25, no. 3, 2008.

[7] G. Nieto, "Biological activities of three essential oils of the lamiaceae family," Medicines, vol. 4, no. 3, p. 63, 2017.

[8] J. Popović-Djordjević, M. Cengiz, M. S. Ozer, and C. Sarikurkcu, "Calamintha incana: essential oil composition and biological activity," Industrial Crops and Products, vol. 128, no. 2018, pp. 162-166, 2019.

[9] G. Çelik, G. Kılıç, and Ş. Kanbolat, "Biological activity, and volatile and phenolic compounds from five Lamiaceae species," Flavour and Fragrance Journal, vol. 36, no. 2, pp. 223-232, 2021.

[10] H. Ouakouak, N. Benchikha, A. Hassani, and M. L. Ashour, "Chemical composition and biological activity of Mentha citrata Ehrh., essential oils growing in southern Algeria," Journal of Food Science and Technology, vol. 56, no. 12, pp. 5346-5353, 2019.

[11] T. R. S. A. Luz, J. A. C. Leite, and L. S. S. de Mesquita, "Seasonal variation in the chemical composition and biological activity of the essential oil of Mesosphaerum suaveolens (L.) Kuntze," Industrial Crops and Products, vol. 153, no. May, Article ID 112600, 2020.

[12] K. Carović-Stanko, M. Petek, and M. Grdiša, "Medicinal plants of the family Lamiaceae as functional foods-a review," Czech Journal of Food Sciences, vol. 34, no. 5, pp. 377-390, 2016.

[13] M. Dhayalan, L. Anitha Jegadeeshwari, and N. Nagendra Gandhi, "Biological activity sources from traditionally usedtribe and herbal plants material," Asian Journal of Pharmaceutical and Clinical Research, vol. 8, no. 6, pp. 11-23, 2015.

[14] C. M. Uritu, C. T. Mihai, and G. D. Stanciu, "Medicinal plants of the family Lamiaceae in pain therapy: A review," Pain Research and Management, vol. 2018, Article ID 7801543, 44 pages, 2018.

[15] J. M. Andrade, C. Faustino, and C. Garcia, "Rosmarinus officinalis L.: an update review of its phytochemistry and biological activity," Future Science $O A$, vol. 4, no. 4, p. FSO283, 2018.

[16] M. Bekut, S. Brkić, N. Kladar, G Dragović, N Gavarić, and B Božin, "Potential of selected Lamiaceae plants in 
anti(retro)viral therapy," Pharmacological Research, vol. 133, pp. 301-314, 2018.

[17] K. P. dos Santos, M. D. Sedano-Partida, and W. R. SalaCarvalho, "Biological activity of Hyptis Jacq. (Lamiaceae) is determined by the environment," Indian Crops Production, vol. 112, pp. 705-715, 2018.

[18] I. Cocan, E. Alexa, and C. Danciu, "Phytochemical screening and biological activity of lamiaceae family plant extracts," Experimental and Therapeutic Medicine, vol. 15, no. 2, pp. 1863-1870, 2018.

[19] L. S. S. d. Mesquita, T. R. S. A. Luz, and J. W. C. d. Mesquita, "Exploring the anticancer properties of essential oils from family Lamiaceae," Food Reviews International, vol. 35, no. 2, pp. 105-131, 2019.

[20] J. O. Pires, P. H. O. Léda, D. R. Oliveira, M. R. CoelhoFerreira, I. S. Scher, and D. M. Talgatti, "Etnobotânica aplicada à seleção de espécies nativas amazônicas como subsídio à regionalização da fitoterapia no SUS: município de Oriximiná - PA, Brasil," Revista Fitos, vol. 14, no. 4, pp. 492-512, 2020.

[21] M. Bahmani, H. Shirzad, M. Majlesi, N. Shahinfard, and M. Rafieian-Kopaei, "A review study on analgesic applications of Iranian medicinal plants," Asian Pacific Journal of Tropical Medicine, vol. 7, no. S1, pp. S43-S53, 2014.

[22] G. Sacchetti, A. Medici, and S. Maietti, "Composition and functional properties of the essential oil of Amazonian basil, Ocimum micranthum Willd., Labiatae in comparison with commercial essential oils," Journal of Agricultural and Food Chemistry, vol. 52, no. 11, pp. 3486-3491, 2004.

[23] G. Lang and G. Buchbauer, "A review on recent research results (2008-2010) on essential oils as antimicrobials and antifungals," Flavour and Fragrance Journal, vol. 27, no. 1, 2012.

[24] F. M. T. Machado and A. Fernandes Junior, Óleos Essenciais: Aspectos Gerais E Usos Em Terapias Naturais, 2011.

[25] T. Kulisic, A. Radonic, V. Katalinic, and M. Milos, "Use of different methods for testing antioxidative activity of oregano essential oil," Food Chemistry, vol. 85, no. 4, 2004.

[26] S. G. Silva, J. N. d. Cruz, and P. L. B. Figueiredo, "Aspectos botânicos dos óleos essenciais," Estudos Transdisciplinares Nas Engenharias, pp. 170-181, 2019.

[27] M. S. de Oliveira, S. G. Silva, and J. N. da Cruz, "Supercritical CO2 application in essential oil extraction," in Industrial Applications of Green Solvents - Volume II, R. M. Inamuddin and A. M. Asiri, Eds., pp. 1-28, Materials Research Foundations, Millersville, PA, USA, 2nd edition, 2019.

[28] M. S. Oliveira, S. Silva, and W. A. Da Costa, Essential Oils Bioactive Compounds, New Perspectives and Applications, 2020.

[29] J. C. Silveira, N. Viégas Busato, A. Oliveira Souza da Costa, and E. Ferreira da Costa Junior, Levantamento E Análise De Métodos De Extração D. E. Óleos Essenciais, 2012.

[30] F. W. F. Bezerra, M. S. de Oliveira, and P. N. Bezerra, "Extraction of bioactive compounds," in Green Sustainable Process for Chemical and Environmental Engineering and Science, A. M. Inamuddin, Asiri, and A. M. Isloor, Eds., pp. 149-167, Elsevier, Amisterdam, Netherlands, 1st edition, 2020.

[31] P. Andrei and A. P. Del Comune, "Aromaterapia e suas aplicações Aromatherapy and its applications," Cadernos, vol. 11, 2005.

[32] M. M. de Christo Scherer, F. M. Marques, and M. M. Figueira, "Wound healing activity of terpinolene and $\alpha$-phellandrene by attenuating inflammation and oxidative stress in vitro," Journal of Tissue Viability, vol. 28, no. 2, pp. 94-99, 2019.

[33] M. Santana de Oliveira, J. N. da Cruz, and W. Almeida da Costa, "Chemical composition, antimicrobial properties of siparuna guianensis essential oil and a molecular docking and dynamics molecular study of its major chemical constituent," Molecules, vol. 25, no. 17, p. 3852, 2020.

[34] M. Santana de Oliveira, V. M. Pereira da Silva, L. Cantão Freitas et al., "Extraction yield, chemical composition, preliminary toxicity of bignonia nocturna (bignoniaceae) essential oil and in silico evaluation of the interaction," Chem. Biodivers.vol. 18, no. 4, 2021.

[35] M. Oussalah, S. Caillet, L. Saucier, and M. Lacroix, "Inhibitory effects of selected plant essential oils on the growth of four pathogenic bacteria: E. coli O157:H7, Salmonella Typhimurium, Staphylococcus aureus and Listeria monocytogenes," Food Control, vol. 18, no. 5, 2007.

[36] L. Gobbo-Neto and N. P. Lopes, "Plantas medicinais: fatores de influência no conteúdo de metabólitos secundários," Química Nova, vol. 30, no. 2, pp. 374-381, 2007.

[37] J. Sharifi-Rad, A. Sureda, and G. Tenore, "Biological activities of essential oils: from plant chemoecology to traditional healing systems," Molecules, vol. 22, no. 1, p. 70, 2017.

[38] L. A. Pupiro Martínez, Y. Pérez Madrugada, and O. Pino Pérez, "Acaricidal activity of essential oils from species belonging to Myrtaceae, Lamiaceae and Rutaceae families against Tetranychus tumidus Banks," Revista de protección vegetal, vol. 33, no. 3, pp. 1-7, 2018.

[39] O. O. Ferreira, J. N. da Cruz, and C. d. J. P. Franco, "First report on yield and chemical composition of essential oil extracted from myrcia eximia DC (Myrtaceae) from the Brazilian Amazon," Molecules, vol. 25, no. 4, p. 783, 2020.

[40] H. J. D. C. Moreira and H. B. N. Bragança, Manual De Identificação de Plantas Infestantes - Hortifruti, Emater e FMC, 2011.

[41] R. M. Harley, "Checklist and key of genera and species of the Lamiaceae of the Brazilian Amazon," Rodriguésia, vol. 63, no. 1, pp. 129-144, 2012.

[42] D. Rivera and Obón, "The ethnobotany of old world Labiatae," in Advances in Labiate Sciences, 1992.

[43] J. A. Kallunki and V. H. Heywood, "Flowering plants of the world," Brittonia, vol. 46, no. 4, 1994.

[44] J. W. Kadereit, Flowering Plants · Dicotyledons, Springer Berlin Heidelberg, Berlin, Heidelberg, 7th edition, 2004.

[45] R. M. Harley, "Notes on the genus gymneia (lamiaceae: ocimeae, hyptidinae) with two new species from Brazil," Phytotaxa, vol. 148, no. 1, p. 57, 2013.

[46] A. S. Soares, J. F. B. Pastore, and J. G. Jardim, "Lamiaceae no rio grande do norte, brasil," Rodriguésia, vol. 70, 2019.

[47] M. C. A. Mota, J. F. B. Pastore, R. Marques Neto, R. M. Harley, and F. R. Salimena, "Lamiaceae na serra negra, minas gerais, brasil," Rodriguésia, vol. 68, no. 1, pp. 143-157, 2017.

[48] J. S. Santos, F. França, M. J. Silva, and M. F. Sales, "Levantamento das espécies de Amasonia (Lamiaceae) para o Brasil," Rodriguésia, vol. 63, no. 4, pp. 1101-1116, 2012.

[49] R. M. Harley, Checklist and key of genera and species of the Lamiaceae of the Brazilian Amazon Lista e chaves para gêneros e espécies de Lamiaceae da Amazônia brasileira, 2012.

[50] H. Lorenzi and F. J. A. Matos, Plantas medicinais no Brasil: nativas e exóticas cultivadas, 2002.

[51] V. A. M. Guerreiro and P. Orientação, Mestrado em Bioquímica Dissertação Mecanismos de Ação Antimicrobiana de Óleos Essenciais de Plantas Condimentares de Família 
Lamiaceae | ESCOLA DE CIÊNCIAS E TECNOLOGIA DEPARTAMENTO DE QUÍMICA, 2018.

[52] R. F. Vieira, R. J. Grayer, A. Patonb, and J. E. Simon, "Uso de marcadores químicos no estudo da diversidade genética de Ocimum gratissimum L," Review Bras Farmacogn.vol. 12, 2002.

[53] R. M. Harley, S. Atkins, and A. L. Budantsev, "Flowering plants dicotyledons: lamiales (except Acanthaceae including Avicenniaceae)," The Families and Genera of Vascular Plants, 2004.

[54] C. M. Uritu, C. T. Mihai, and G.-D. Stanciu, "Medicinal plants of the family lamiaceae in pain therapy: a review," Pain Research Management, vol. 2018, Article ID 7801543, 44 pages, 2018.

[55] M. A. C. Freitas, A. V. Amorim, and A. M. E. Bezerra, "Crescimento e tolerância à salinidade em três espécies medicinais do gênero Plectranthus expostas a diferentes níveis de radiação," Review Bras. Plantas Medicine, vol. 16, no. 4, 2014.

[56] E. J. Crevelin, S. C. Caixeta, and H. J. Dias, "Antimicrobial activity of the essential oil of plectranthus neochilus against cariogenic bacteria," Evidence-Based Complementary Alternative Medicine, vol. 2015, Article ID 102317, 6 pages, 2015.

[57] G. P. Aguiar, K. A. Lima, and Marcela, "Antifungal activity of the essential oils of plectranthus neochilus (Lamiaceae) and tagetes erecta (Asteraceae) cultivated in Brazil," International Journal of Complementary Alternative Medicine, vol. 11, no. 2, 2018.

[58] S. C. Caixeta, L. G. Magalhães, and N. I. de Melo, "Chemical composition and in vitro schistosomicidal activity of the essential oil of plectranthus neochilus grown in Southeast Brazil," Chemistry \& Biodiversity, vol. 8, no. 11, pp. 49-57, 2011.

[59] E. L. L. Baldin, A. E. M. Crotti, and K. A. L. Wakabayashi, "Plant-derived essential oils affecting settlement and oviposition of Bemisia tabaci (Genn.) biotype B on tomato," Journal of Pest Science, vol. 86, no. 2, 2013.

[60] T. L. Fanela, E. L. Baldin, and L. E. Pannuti, "Lethal and inhibitory activities of plant-derived essential oils against Bemisia tabaci gennadius (Hemiptera: aleyrodidae) biotype B in tomato," Neotropical Entomology, vol. 45, no. 2, pp. 201-10, 2016.

[61] Y.S. Kuo, H. F. Chien, and W. Lu, "Plectranthus amboinicus and Centella asiatica cream for the treatment of diabetic foot ulcers," Evidence-based Complementary Alternative Medicine, vol. 2012, Article ID 418679, 10 pages, 2012.

[62] M. Khosla, "Study of inter-relationship, phylogeny and evolutionary tendencies in genus Ocimum," Indian Journal of Genetic Plant Breedings, vol. 55, no. 1, 1995.

[63] U. P. Albuquerque and L. H. C. Andrade, Dialnet-El Genero Ocimum Lamiaceae En El Nordeste Del Brasil-70509, 1998.

[64] U. P. de Albuquerque, "Three new varieties in Ocimum L. (Lamiaceae)," Brazilian Archives of Biology and Technology, vol. 42, no. 1, 1999.

[65] R. F. Vieira and J. E. Simon, "Chemical characterization of basil (Ocimum spp.) found in the markets and used in traditional medicine in Brazil," Economic Botany, vol. 54, no. $2,2000$.

[66] H. Lorenzi and F. J. de A. Matos, "Plantas medicinais no Brasil: nativas e exóticas, Odessa Instant Plant., 2008.

[67] J. C. Nascimento, L. C. Barbosa, and V. F. Paula, "Chemical composition and antimicrobial activity of essential oils of Ocimum canum Sims. and Ocimum selloi Benth," Anais da
Academia Brasileira de Ciencias, vol. 83, no. 3, pp. 787-99, 2011.

[68] M. Govindarajan, R. Sivakumar, M. Rajeswary, and K. Yogalakshmi, "Chemical composition and larvicidal activity of essential oil from Ocimum basilicum (L.) against Culex tritaeniorhynchus, Aedes albopictus and Anopheles subpictus (Diptera: Culicidae)," Experimental Parasitology, vol. 134, no. 1, pp. 7-11, 2013.

[69] L. Scalvenzi, M. Radice, and L. Toma, "Larvicidal activity of Ocimum campechianum, Ocotea quixos and Piper aduncum essential oils against Aedes aegypti," Parasite (Paris, France), vol. 26, p. 23, 2019.

[70] L. P. Ricarte, G. P. Bezerra, and N. R. Romero, "Chemical composition and biological activities of the essential oils from Vitex-agnus castus, Ocimum campechianum and Ocimum carnosum," Anais da Academia Brasileira de Ciencias, vol. 92, no. 1, pp. 9-11, 2020.

[71] C. Y. Wang, S. Y. Wang, J. J. Yin, J. Parry, and L. L. Yu, "Enhancing antioxidant, antiproliferation, and free radical scavenging activities in strawberries with essential oils," Journal of Agricultural and Food Chemistry, vol. 55, no. 16, pp. 27-32, 2007.

[72] C. V. Nakamura, T. Ueda-Nakamura, E. Bando, A. F Melo, D. A Cortez, and B. P Dias Filho, "Antibacterial activity of Ocimum gratissimum L. Essential oil," Memorias Do Instituto Oswaldo Cruz, vol. 94, no. 5, pp. 5-8, 1999.

[73] R. S. Pereira, T. C. Sumita, M. R. Furlan, A. O. C. Jorge, and M. Ueno, "Atividade antibacteriana de óleos essenciais em cepas isoladas de infecção urinária," Rev. Saude Publica, vol. 38, no. 2, 2004.

[74] D. Falcão and F. Menezes, "Revisão etnofarmacológica, farmacológica e química do gênero Hyptis," Review Bras. Farm, vol. 84, no. 3, 2003.

[75] G. O. Onawunmi and E. O. Ogunlana, "A study of the antibacterial activity of the essential oil of lemon grass (cymbopogon citratus (DC.) stapf)," Pharmaceutical Biology, vol. 24 , no. 2, 1986.

[76] C. X. You, K. Yang, and Y. Wu, "Chemical composition and insecticidal activities of the essential oil of Perilla frutescens (L.) Britt. aerial parts against two stored product insects," European Food Research and Technology, vol. 239, no. 3, pp. 481-490, 2014.

[77] P. Tiwari, "Recent advances and challenges in trichome research and essential oil biosynthesis in Mentha arvensis L," Industrial Crops and Products, vol. 82, pp. 141-148, 2016.

[78] B. Salehi, Z. Stojanović-Radić, and J. Matejić, "Plants of genus Mentha: from farm to food factory," Plants, vol. 7, no. 3, p. 70, 2018.

[79] F. Z. Benomari, V. Andreu, and J. Kotarba, "Essential oils from Algerian species of Mentha as new bio-control agents against phytopathogen strains," Environmental Science and Pollution Research, vol. 25, no. 30, pp. 29889-29900, 2018.

[80] F. Anwar, A. Abbas, T. Mehmood, A. H. Gilani, and N. u. Rehman, "Mentha: a genus rich in vital nutra-pharmaceuticals-A review," Phytotherapy Research, vol. 33, no. 10, pp. 2548-2570, 2019.

[81] P. Singh and A. K. Pandey, "Prospective of essential oils of the genus Mentha as biopesticides: a review," Frontiers in Plant Science, vol. 9, no. Sep, p. 1295, 2018.

[82] L. M. P. C. Madureira, Cancela, and P. Doutora, Avaliação da composição química, atividade antioxidante e atividade antimicrobiana de Segurelha (Satureja montana) Orientado por, 2020. 
[83] M. C. I. Navroski, D. A. G. Waldow, and L. R. S. Reiniger, "Multiplicação in vitro de segmentos apicais caulinares de segurelha (Satureja hortensis L.)," Revista Brasileira de Plantas Medicinais, vol. 16, no. 1, 2014.

[84] G. Miguel, M. Simões, and A. C. Figueiredo, "Composition and antioxidant activities of the essential oils of Thymus caespititius, Thymus camphoratus and Thymus mastichina," Food Chem.vol. 86, no. 2, 2004.

[85] M. V. Beloni, M. Aparecida Da Silva, and L. Oliveira, "Atividade antibacteriana dos óleos essenciais frente a agentes causadores da mastite bovina," in Tópicos Especiais Em Ciência Animal IX, p. 262, 2020.

[86] O. Koul, R. Singh, B. Kaur, and D. Kanda, "Comparative study on the behavioral response and acute toxicity of some essential oil compounds and their binary mixtures to larvae of Helicoverpa armigera, Spodoptera litura and Chilo partellus," Industrial Crops and Products, vol. 49, pp. 428-436, 2013.

[87] N. Cárdenas-Ortega, M. González-Chávez, and R. FigueroaBrito, "Composition of the essential oil of Salvia ballotiflora (lamiaceae) and its insecticidal activity," Molecules, vol. 20, no. 5, pp. 8048-8059, 2015.

[88] S. Grausgruber-Gröger, C. Schmiderer, R. Steinborn, and J. Novak, "Seasonal influence on gene expression of monoterpene synthases in Salvia officinalis (Lamiaceae)," Journal of Plant Physiology, vol. 169, no. 4, pp. 353-359, 2012.

[89] C. V. V. Castilho, R. R. Fantatto, and Y. A. Gaínza, "In vitro activity of the essential oil from Hesperozygis myrtoides on Rhipicephalus (Boophilus) microplus and Haemonchus contortus," Revista Brasileira de Farmacognosia, vol. 27, no. 1, pp. 70-76, 2017.

[90] E. C. Chagas, C. Majolo, and P. C. Monteiro, "Composition of essential oils ofMenthaspecies and their antimicrobial activity againstAeromonasspp," Journal of Essential Oil Research, vol. 32, no. 3, pp. 209-215, 2020.

[91] F. C. Rodrigues, J. W. Almeida-Bezerra, and K. R. Fidelis, "Chemical characterization and insecticidal potential of the essential oil of Ocimum gratissimum L. (Lamiaceae) against Nauphoeta cinerea (Blaberidae)," Research, Society and Development, vol. 9, no. 9, Article ID e72996412, 2020.

[92] L. G. Osório, A. L. Silva, and A. O. S. Fonseca, "Atividade in vitro do óleo essencial de Origanum vulgare L. em isolados clínicos de Aspergillus spp," Arquivo brasileiro de medicina veterinária e zootecnia, vol. 71, no. 1, pp. 204-210, 2019.

[93] H. C. Passinho-Soares, J. P. David, and J. R. F. d. Santana, "Influence of growth regulators on distribution of trichomes and the production of volatiles in micropropagated plants of Plectranthus ornatus," Revista Brasileira de Farmacognosia, vol. 27, no. 6, pp. 679-690, 2017.

[94] A. Giatropoulos, A. Kimbaris, and A. Michaelakis, "Chemical composition and assessment of larvicidal and repellent capacity of 14 Lamiaceae essential oils against Aedes albopictus," Parasitol. Res.vol. 117, no. 6, pp. 1953-1964, 2018.

[95] R. Lopes Martins, A. Bruno Lobato Rodrigues, and É. de Menezes Rabelo, "Development of larvicide nanoemulsion from the essential oil of Aeollanthus suaveolens Mart. ex Spreng against Aedes aegypti, and its toxicity in nontarget organism," Arabian Journal of Chemistry, vol. 14, no. 6, Article ID 103148, 2021.

[96] M. Božović and R. Ragno, "Calamintha nepeta (L.) savi and its main essential oil constituent pulegone: biological activities and chemistry," Molecules (Basel, Switzerland), vol. 22, no. 2, p. 290, 2017.
[97] A. Rojas-Olivos, R. Solano-Gómez, and C. GranadosEchegoyen, "Larvicidal effect of Clinopodium macrostemum essential oil extracted by microwave-assisted hydrodistillation against Culex quinquefasciatus (Diptera: Culicidae)," Revista da Sociedade Brasileira de Medicina Tropical, vol. 51, no. 3, pp. 291-296, 2018.

[98] S. P. Almeida, A. A. M. de Filho, and F. G. Simplicio, "Chemical profile, toxicity, anti-acetylcholinesterase and antimicrobial activity of essential oil from hyptis dilatata leaves," Chem. Eng. Trans.vol. 64, pp. 271-276, 2018.

[99] A. G. R. Barbosa, C. D. M. Oliveira, and L. J. Lacerda-Neto, "Evaluation of chemical composition and antiedematogenic activity of the essential oil of Hyptis martiusii Benth," Saudi Journal of Biological Sciences, vol. 24, no. 2, pp. 355-361, 2017.

[100] B. Justus, V. P. de Almeida, and M. M. Gonçalves, “"Chemical composition and biological activities of the essential oil and anatomical markers of Lavandula dentata L. Cultivated in Brazil," Brazilian arch," Biology and Technology, vol. 61, 2018.

[101] H. Niksic, K. Duric, and K. Duric, "In vitro antiproliferative activity of Melissa officinalis L (Lamiaceae) leaves essential oil," Boletin Latinoamericano y del Caribe de Plantas Medicinales y Aromaticas, vol. 18, no. 5, pp. 480-491, 2019.

[102] L. C. da Silva, W. M. de Souza Perinotto, and F. A. Sá, "In vitro acaricidal activity of Cymbopogon citratus, Cymbopogon nardus and Mentha arvensis against Rhipicephalus microplus (Acari: ixodidae)," Experimental Parasitology, vol. 216, Article ID 107937, 2020.

[103] F. R. Brandão, C. F. S. Farias, and D. C. de Melo Souza, "Anesthetic potential of the essential oils of Aloysia triphylla, Lippia sidoides and Mentha piperita for Colossoma macropomum," Aquaculture, vol. 534, Article ID 736275, 2021.

[104] J. Benites, A. Guerrero-Castilla, and F. Salas, "Chemical composition, in vitro cytotoxic and antioxidant activities of the essential oil of Peruvian minthostachys mollis Griseb," Boletin Latinoamericano y del Caribe de Plantas Medicinales y Aromaticas, vol. 17, no. 6, pp. 566-574, 2018.

[105] D. Dris, F. Tine-Djebbar, H. Bouabida, and N. Soltani, "Chemical composition and activity of an Ocimum basilicum essential oil on Culex pipiens larvae: toxicological, biometrical and biochemical aspects," South African Journal of Botany, vol. 113, pp. 362-369, 2017.

[106] P. T. Miura, S. C. N. Queiroz, C. M. Jonsson, E. C. Chagas, F. C. M. Chaves, and F. G. Reyes, "Study of the chemical composition and ecotoxicological evaluation of essential oils in Daphnia magna with potential use in aquaculture," Aquaculture Research, vol. 52, no. 7, pp. 3415-3424, 2021.

[107] M. Govindarajan, S. Kadaikunnan, N. S. Alharbi, and G. Benelli, "Acute toxicity and repellent activity of the Origanum scabrum Boiss. \& Heldr. (Lamiaceae) essential oil against four mosquito vectors of public health importance and its biosafety on non-target aquatic organisms," Environmental Science and Pollution Research, vol. 23, no. 22, pp. 23228-23238, 2016.

[108] A. P. P. Mota, J. C. P. Dantas, and C. C. Frota, "Antimicrobial activity of essential oils from Lippia alba, Lippia sidoides, Cymbopogon citrates, Plectranthus amboinicus, and Cinnamomum zeylanicum against Mycobacterium tuberculosis," Ciência Rural, vol. 48, no. 6, 2018.

[109] F. A. R. Alves, S. M. de Morais, and A. C. N. Sobrinho, "Chemical composition, antioxidant and antifungal activities of essential oils and extracts from Plectranthus spp. against dermatophytes fungi," Rev. Bras. Saúde e Produção Anim.vol. 19, no. 1, pp. 105-115, 2018. 
[110] N. Barbieri, M. Costamagna, and M. Gilabert, “Antioxidant activity and chemical composition of essential oils of three aromatic plants from la Rioja province," Pharmaceutical Biology, vol. 54, no. 1, pp. 168-173, 2016.

[111] A. Yashin, Y. Yashin, X. Xia, and B. Nemzer, “Antioxidant activity of spices and their impact on human health: a review," Antioxidants (Basel, Switzerland), vol. 6, no. 3, pp. 1-18, 2017.

[112] D. Gupta, "Methods for determination of antioxidant capacity: a review," International Journal of Pharmaceutical Sciences and Research, vol. 6, no. 2, pp. 546-566, 2015.

[113] L. A. da Silva, J. D. A. Raposo, and L. P. G. Campos, "Atividade antioxidante do óleo essencial de Myrcia sylvatica (G. Mey.) DC. por diferentes métodos de análises antioxidantes (ABTS, DPPH, FRAP, $\beta$-caroteno/ácido linoleico)," Review Fitos, vol. 12, no. 2, pp. 117-126, 2018.

[114] C. Zorzetto, C. C. Sánchez-Mateo, and R. M. Rabanal, "Antioxidant activity and cytotoxicity on tumour cells of the essential oil fromCedronella canariensisvar.canariensis(L.) Webb \& Berthel. (Lamiaceae)," Natural Product Research, vol. 29, no. 17, pp. 1641-1649, 2015.

[115] R. Singh, M. A. M. Shushni, and A. Belkheir, "Antibacterial and antioxidant activities of Mentha piperita L," Arabian Journal of Chemistry, vol. 8, no. 3, pp. 322-328, 2015.

[116] A. Bouyahya, A. Et-Touys, and Y. Bakri, "Chemical composition of Mentha pulegium and Rosmarinus officinalis essential oils and their antileishmanial, antibacterial and antioxidant activities," Microbial Pathogenesis, vol. 111, pp. 41-49, 2017.

[117] B. Fatiha, M. Khodir, and D. Nabila, "Assessment of the chemical composition andin vitroAntioxidant activity ofMentha rotundifolia(L.) huds essential oil from Algeria," Journal of Essential Oil Bearing Plants, vol. 19, no. 5, pp. 1251-1260, 2016.

[118] S. K. Bardaweel, B. Bakchiche, and H. A. ALSalamat, "Chemical composition, antioxidant, antimicrobial and Antiproliferative activities of essential oil of Mentha spicata L. (Lamiaceae) from Algerian Saharan atlas," BMC Complementary and Alterntive Medicine, vol. 18, no. 1, pp. 1-7, 2018.

[119] H. Niksic, K. Duric, and E. Omeragic, "Chemical characterization, antimicrobial and antioxidant properties of Mentha spicata L. ( Lamiaceae ) essential oil," Bull. Chem. Tehcnologists Bosnia Herzegovina, vol. 50, pp. 43-48, 2018.

[120] A. Farouk, R. Fikry, and M. Mohsen, "Chemical composition and antioxidant activity ofOcimum basilicumL. Essential oil cultivated in madinah monawara, Saudi arabia and its comparison to the Egyptian chemotype," Journal of Essential Oil Bearing Plants, vol. 19, no. 5, pp. 1119-1128, 2016.

[121] G. Mitropoulou, E. Fitsiou, and E. Stavropoulou, "Composition, antimicrobial, antioxidant, and antiproliferative activity of Origanum dictamnus (dittany) essential oil," Microbial Ecology in Health and Disease, vol. 26, no. 0, p. 26543, 2015.

[122] N. Hadjadj and M. Hazzit, "Analysis and antioxidant activity of essential oils and methanol extracts of Origanum floribundum munby," Journal of Essential Oil Bearing Plants, vol. 23, no. 1, pp. 85-96, 2020.

[123] J. R. N. Santos, A. M. Teles, C. G. Ferreira, and A. N. Mouchrek, "Avaliação da atividade bactericida e antioxidante do óleo essencial e do extrato hidroalcoólico de orégano (Origanum vulgare)," Research, Society and Development, vol. 9, no. 10, Article ID e7829108410, 2020.
[124] M. R. Morshedloo, H. Mumivand, L. E. Craker, and F. Maggi, "Chemical composition and antioxidant activity of essential oils in Origanum vulgare subsp. gracile at different phenological stages and plant parts," Journal of Food Process Preservative, vol. 42, no. 2, pp. 1-8, 2018.

[125] S. Gezici, N. Sekeroglu, and A. Kijjoa, "In vitro anticancer activity and antioxidant properties of essential oils from Populus alba L. and Rosmarinus officinalis L. from South Eastern Anatolia of Turkey," Indian Journal Pharmacy and Educational Research, vol. 51, no. 3, pp. S498-S503, 2017.

[126] A. R. Ladan Moghadam, "Antioxidant activity and chemical composition ofRosmarinus officinalisL. Essential oil from Iran," Journal of Essential Oil Bearing Plants, vol. 18, no. 6, pp. 1490-1494, 2015.

[127] A. R. Ladan Moghadam, "Antioxidant activity and essential oil evaluation of Satureja hortensis L. ( lamiaceae ) from Iran," Journal of Essential Oil Bearing Plants, vol. 18, no. 2, pp. 455-459, 2015.

[128] N. Z. Mamadalieva, F. Sharopov, P. Satyal, S. S. Azimova, and M. Wink, "Composition of the essential oils of three Uzbek Scutellaria species (Lamiaceae) and their antioxidant activities," Natural Product Research, vol. 31, no. 10, pp. 1172-1176, 2017.

[129] S. Hammami, R. El Mokni, and K. Faidi, "Chemical composition and antioxidant activity of essential oil from aerial parts of Teucrium flavum L. subsp. flavum growing spontaneously in Tunisia," Natural Product Research, vol. 29, no. 24, pp. 2336-2340, 2015.

[130] M. B. Goudjil, S. Zighmi, D. Hamada, Z. Mahcene, S. E. Bencheikh, and S. Ladjel, "Biological activities of essential oils extracted from Thymus capitatus (Lamiaceae)," South African Journal of Botany, vol. 128, pp. 274-282, 2020.

[131] L. Diniz Do Nascimento, A. A. B. Moraes, and K. S. Costa, "Bioactive natural compounds and antioxidant activity of essential oils from spice plants: new findings and potential applications," Biomolecules, vol. 10, no. 7, p. 988, 2020.

[132] M. Bonesi, M. R. Loizzo, R. Acquaviva, G. A Malfa, F Aiello, and $\mathrm{R}$ Tundis, "Anti-inflammatory and antioxidant agents from Salvia genus (lamiaceae): an assessment of the current state of knowledge," Anti-inflammatory \& Anti-allergy Agents in Medicinal Chemistry, vol. 16, no. 2, pp. 70-86, 2017.

[133] R. S. Borges, B. L. S. Ortiz, A. C. M. Pereira, H. Keita, and J. C. T. Carvalho, "Rosmarinus officinalis essential oil: a review of its phytochemistry, anti-inflammatory activity, and mechanisms of action involved," Journal of Ethnopharmacology, vol. 229, pp. 29-45, 2019.

[134] R. R. Simões, I. D. Coelho, and S. C. Junqueira, "Oral treatment with essential oil of Hyptis spicigera Lam. (Lamiaceae) reduces acute pain and inflammation in mice: potential interactions with transient receptor potential (TRP) ion channels," Journal of Ethnopharmacology, vol. 200, pp. 8-15, 2017.

[135] W. Luo, Z. Du, and Y. Zheng, "Phytochemical composition and bioactivities of essential oils from six Lamiaceae species," Industrial Crops and Products, vol. 133, pp. 357-364, 2019.

[136] W. Abdelli, F. Bahri, and A. Romane, "Chemical composition and anti-inflammatory activity of algerian thymus vulgaris essential oil," Natural Product Communications, vol. 12, no. 4, pp. 611-614, 2017.

[137] R. Avola, G. Granata, C. Geraci, E. Napoli, A. C. E. Graziano, and V. Cardile, "Oregano (Origanum vulgare L.) essential oil provides anti-inflammatory activity and facilitates wound healing in a human keratinocytes cell model," Food and 
Chemical Toxicology, vol. 144, no. May, Article ID 111586 , 2020.

[138] R. S. S. Barreto, J. S. S. Quintans, and R. K. L. Amarante, "Evidence for the involvement of TNF- $\alpha$ and IL-1 $\beta$ in the antinociceptive and anti-inflammatory activity of Stachys lavandulifolia Vahl. (Lamiaceae) essential oil and (-)- $\alpha$-bisabolol, its main compound, in mice," Journal of Ethnopharmacology, vol. 191, pp. 9-18, 2016.

[139] A. K. Ruiters, P. M. Tilney, S. F. Van Vuuren, A. M. Viljoen, G. P. P. Kamatou, and B.-E. Van Wyk, "The anatomy, ethnobotany, antimicrobial activity and essential oil composition of southern African species of Teucrium (Lamiaceae)," South African Journal of Botany, vol. 102, pp. 175-185, 2016.

[140] Y. Laghmouchi, O. Belmehdi, N. S. Senhaji, and J. Abrini, "Chemical composition and antibacterial activity of Origanum compactum Benth. essential oils from different areas at northern Morocco," South African Journal of Botany, vol. 115, pp. 120-125, 2018.

[141] Z. Jalal, Y. El Atki, B. Lyoussi, and A. Abdellaoui, "Phytochemistry of the essential oil of Melissa officinalis L. growing wild in Morocco: preventive approach against nosocomial infections," Asian Pacific Journal of Tropical Biomedicine, vol. 5, no. 6, pp. 458-461, 2015.

[142] M. Khan, S. T. Khan, N. A. Khan, A. Mahmood, A. A. AlKedhairy, and H. Z. Alkhathlan, "The composition of the essential oil and aqueous distillate of Origanum vulgare L. growing in Saudi Arabia and evaluation of their antibacterial activity," Arabian Journal of Chemistry, vol. 11, no. 8, pp. 1189-1200, 2018.

[143] G. Schött, S. Liesegang, and F. Gaunitz, "The chemical composition of the pharmacologically active Thymus species, its antibacterial activity against Streptococcus mutans and the antiadherent effects of T. vulgaris on the bacterial colonization of the in situ pellicle," Fitoterapia, vol. 121, no. May, pp. 118-128, 2017.

[144] A. Alimpić, D. Pljevljakušić, and K. Šavikin, "Composition and biological effects of Salvia ringens (Lamiaceae) essential oil and extracts," Indian Crops Production, vol. 76, pp. 702-709, 2015.

[145] S.-K. Yang, K. Yusoff, and W. Thomas, "Lavender essential oil induces oxidative stress which modifies the bacterial membrane permeability of carbapenemase producing Klebsiella pneumoniae," Scientific Reports, vol. 10, no. 1, p. 819, 2020.

[146] X. Wang, Y. Shen, and K. Thakur, "Antibacterial activity and mechanism of ginger essential oil against Escherichia coli and Staphylococcus aureus," Molecules, vol. 25, no. 17, p. 3955, 2020.

[147] A. Skendi, D. N. Katsantonis, P. Chatzopoulou, M. Irakli, and M. Papageorgiou, "Antifungal activity of aromatic plants of the lamiaceae family in bread," Foods, vol. 9, no. 11, pp. 8-12, 2020.

[148] J. Ramírez, G. Gilardoni, and M. Jácome, "Chemical composition, Enantiomeric analysis, AEDA sensorial evaluation and antifungal activity of the essential oil from the Ecuadorian plant Lepechinia mutica Benth (lamiaceae)," Chemistry \& Biodiversity, vol. 14, no. 12, Article ID e1700292, Dec. 2017.

[149] C. Rus, R. M. Sumalan, and E. Alexa, "Study on chemical composition and antifungal activity of essential oils obtained from representative species belonging to the Lamiaceae family," Plant and Soil Environment, vol. 61, no. 7, pp. 297-302, 2015.
[150] D. Neveen, H. A. Mohamed, L. A. El-Kassem, and M. Khalil, "Chemical composition and antifungal activity of Syzygium aromaticum L. essential oil," Iran. Journal of Medicinal Aromatic Plants, vol. 33, no. 4, pp. 552-561, 2017.

[151] A. Piras, M. J. Gonçalves, and J. Alves, "Ocimum tenuiflorum L. and Ocimum basilicum L., two spices of Lamiaceae family with bioactive essential oils," Indian Crops Production, vol. 113, pp. 89-97, 2018.

[152] A. Kumar and V. B. Kudachikar, "Antifungal properties of essential oils against anthracnose disease: a critical appraisal," Journal of Plant Disorder Protection, vol. 125, pp. 133-144, 2017. 